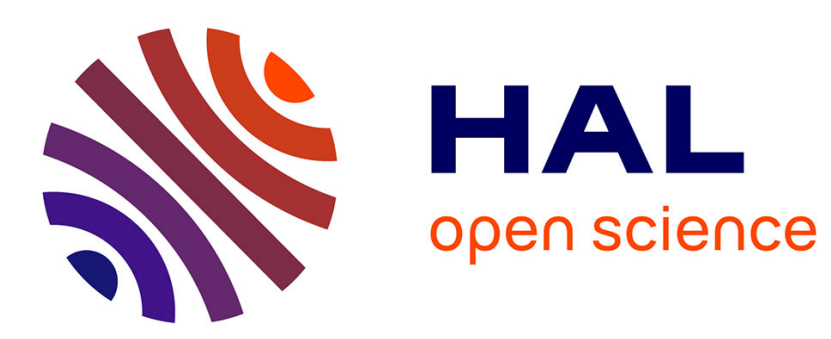

\title{
Stacking sequence and shape optimization of laminated composite plates via a level-set method \\ Grégoire Allaire, Gabriel Delgado
}

\section{To cite this version:}

Grégoire Allaire, Gabriel Delgado. Stacking sequence and shape optimization of laminated composite plates via a level-set method. Journal of the Mechanics and Physics of Solids, 2016, 97, pp.168-196. hal-01164929v2

\section{HAL Id: hal-01164929 \\ https://hal.science/hal-01164929v2}

Submitted on 1 Jul 2016

HAL is a multi-disciplinary open access archive for the deposit and dissemination of scientific research documents, whether they are published or not. The documents may come from teaching and research institutions in France or abroad, or from public or private research centers.
L'archive ouverte pluridisciplinaire HAL, est destinée au dépôt et à la diffusion de documents scientifiques de niveau recherche, publiés ou non, émanant des établissements d'enseignement et de recherche français ou étrangers, des laboratoires publics ou privés. 


\title{
Stacking sequence and shape optimization of laminated composite plates via a level-set method
}

\author{
G. Allaire ${ }^{1}$, G. Delgado ${ }^{2}$, \\ July 1, 2016 \\ ${ }^{1}$ CMAP, UMR CNRS 7641, Ecole Polytechnique, 91128 Palaiseau. \\ ${ }^{2}$ IRT SystemX, Palaiseau, France.
}

\begin{abstract}
We consider the optimal design of composite laminates by allowing a variable stacking sequence and in-plane shape of each ply. In order to optimize both variables we rely on a decomposition technique which aggregates the constraints into one unique constraint margin function. Thanks to this approach, an exactly equivalent bi-level optimization problem is established. This problem is made up of an inner level represented by the combinatorial optimization of the stacking sequence and an outer level represented by the topology and geometry optimization of each ply. We propose for the stacking sequence optimization an outer approximation method which iteratively solves a set of mixed integer linear problems associated to the evaluation of the constraint margin function. For the topology optimization of each ply, we lean on the level set method for the description of the interfaces and the Hadamard method for boundary variations by means of the computation of the shape gradient. Numerical experiments are performed on an aeronautic test case where the weight is minimized subject to different mechanical constraints, namely compliance, reserve factor and buckling load.
\end{abstract}

Keywords: Composite laminates; Stacking sequence; Topology optimization; Level-set method; Decomposition.

\section{Introduction}

Structural optimization in aeronautics usually looks for the lightest structure which sustains the forces and environmental conditions that an aircraft will typically find during operation. Within this framework, frequently the objective function (total mass) is easy to evaluate if a good parametrization of the structure is available, meanwhile the mechanical constraints are in general much more complicated to evaluate. It requires a mechanical model which can be time-consuming to solve, depending on the choice of variables. Classically, this optimization process has been done by the engineer expertise. However, the increment of the size of the design space does not allow to find the best design without automatizing the process. As a response to this challenge, several techniques for size, shape and topology optimization have successfully been developed and applied to aircraft design [6, 11, 30, 31, 57, 68, 69.

During the last years, a special type of material has become quite popular in automotive and aerospace industries: multi-layered composites. These materials benefit from very attractive features such as low weight, high fatigue resistance and good endurance against corrosion and other harsh environmental conditions. The properties of multi-layered composite structures strongly depend on the shape, the orientation of the reinforcement and the stacking sequence of the laminate. Indeed the directional nature of the fibers in a fiber-reinforced laminate introduces directional dependence of the strength, thermal and electrical conductivity. Meanwhile the stacking sequence has a strong influence on the bending behavior of the laminate.

In view of the increasing use of composite materials within industry, their optimal design has drawn great attention of the scientific community. We refer e.g. to Gürdal, Haftka and co-workers 4, 30, 31, 42, 68, 70, 71, but also to [41, 44, 55, 56. Actually, composite materials possess a large number of design possibilities which makes their optimization a complex problem. A typical composite laminate may be characterized by design variables which are continuous (geometry, size of the structure, material distribution in each ply) or discrete (orientation of the fibers, lay-up or stacking sequence). Additionally, when designing composite aircraft structural components, one must take into account constraints on the 
structural performance (accelerations, buckling factors, displacement, material failure criteria, etc.) and equally constraints on the global and local manufacturing rules imposed during the composite manufacturing process. These constraints are specific to the type of technology used and industrial policies (symmetric and balanced laminates, ply drops and overlaps, etc.). A particularly difficult constraint to deal with is structural integrity or blending, i.e., the continuity of the stacking sequence across multiple panels making up the structure [3, 66, 78,

The large number of design variables and the complex relationship between these variables and the output performance, make composite structure design extremely challenging. Decomposition procedures are appealing approaches to treat such a complexity in composite optimization. A typical decomposition/bilevel scheme aims to break up the large problem into many problems that matches the different levels of analysis taking into account its interactions during the design process. In the case of composite design, the goal of this technique is to combine a "continuous medium" vision, necessary for the overall understanding of the phenomena related to the stiffness level, and the discrete nature of laminated composites. For example a continuous representation of the composite is applied at the system level (fuselage or wing), meanwhile the subsystem level (lay-up of the panel) is treated with discrete algorithms. For more examples we refer the reader to [10, 12, 18, 46, 48, 49, 50, 74. There are other decomposition approaches in the literature, which are not necessarily based on the notion of lamination parameters, as in [1], 2], 52], 53.

Once the problem is decomposed, the optimal design of the composite lay-up or stacking sequence emerges naturally as a mixed integer non linear programming problem, for which many methods exist in the literature. Some of them are deterministic, e.g. the application of the branch and bound method for minimal mass problems with a buckling constraint [30, 32, 59, or the benders decomposition method for the optimization of tailored fiber orientation composites [58. These methods guarantee in general to find a global solution provided that the problem satisfies certain conditions such as convexity. When no such conditions are satisfied, an efficient complement and/or alternative to deterministic methods are heuristics: e.g. the penalty function approach [17, 75] and a rounding procedure using lamination parameters [37, 38, 76]. Alternatively, one can rely on so-called stochastic methods which, in the literature, are mostly genetic algorithms [4, 42, 47, 60, 70, 71, with only a few exceptions (see e.g. [26] for simulated annealing).

The previous approaches for the combinatorial optimization of the stacking sequence have proved effective, but they are usually decoupled from the detailed and pointwise description of each layer. In truth, the design of the in-plane shape of each layer has been much less considered so far, despite of its importance in terms of structural strength and manufacturability. The in-plane shape as a design variable can only be treated satisfactorily through a continuous approach, which leads to reconsider the former continuous/discrete framework. There are however a few works on topology optimization applied to fiber orientation tailoring of laminated composites, carried out by Lund and his co-workers [39, 45, 51, 61, 75] and also by [16, 22. Most of these works rely on a density-based method (Solid Isotropic Material with Penalization or SIMP [11, [14, Discrete Material Optimization or DMO [75], 73]). This approach has also been extended to take into account robustness [35], manufacturing constraints [72] and eigenfrequency constraints [13].

The present article addresses the structural optimization of a composite laminate by adding a new design variable to the fiber orientation and lay-up sequence: the in-plane shape and topology of each ply. Indeed we suppose that each ply is made up of two phases (one of them being void) and the position of the interface is the design variable. When add up several layers, the composite laminate constitutes a multi-material. To the best of our knowledge, this approach is relatively novel and very few industrial design tools show similar features. Moreover the problem we contemplate to solve is different of the fiber orientation tailoring problem mentioned above, since the fiber orientation within each phase remains constant. A similar approach, with a completely different numerical method, can be found in the composite optimization routine of the Altair ${ }^{\circledR}$ software OptiStruct ${ }^{\circledR}[29,82$.

For the topology design of each ply, we rely on the level-set approach for multi-phase optimization detailed in [7]. First introduced in [63], the level-set method has the advantage of tracking the interfaces on a fixed mesh, easily managing topological changes without any need of re-meshing. Allied to the Hadamard method of shape differentiation, the level-set approach is an efficient shape and topology optimization algorithm [8, 80, which gives a better description and control of the geometrical properties of the interface without need of any intermediate density, as most of popular algorithm do [11, avoiding typical drawbacks such as intermediate density penalization and possible spurious physical behavior during the optimization process.

Due to the mixed character of the composite optimization problem (continuous shape and discrete 
stacking sequence), another essential ingredient is a decomposition technique which splits the problem into different levels. On the one hand, the optimization is carried in each ply by a level-set method to find its optimal shape. On the other hand, the combinatorial optimization of the stacking sequence is performed by an outer approximation method [25. It is a deterministic method which is exact for convex functions and consists in iteratively approximating the objective and constraints of the problem by linear under-estimators. Thanks to a careful choice of the parametrization of the stacking sequence, the number of discrete variables is moderate, which makes such classical linear integer programming-based solvers very efficient and competitive with respect to the most popular heuristics and stochastic methods (which suffer from high computational cost).

This article is organized as follows. In Section 2 the composite multi-layered optimization problem is formulated. It is a mixed optimization approach in the sense that there are two optimization variables: one discrete (the stacking sequence), the other continuous (the ply shapes). We thus rely on a decomposition framework and on the notion of a constraint margin function for its numerical optimization. The stacking sequence optimization is detailed in Section 3. The shape optimization of each ply is explained in Section 4 In particular, we recall the notion of Hadamard shape derivative (which, here, is rather a derivative with respect to an interface) and the level set approach to describe the shape of each ply inside the laminated composite plate. Finally numerical examples for three different types of constraints are given in Section 5 . Our numerical test cases are quite simplified to focus on the main difficulties but are representative of real aeronautic cases in an early design phase. One important simplification is that the composite panel is flat so we use a plate model rather than a shell model with ribs. Our numerical examples are very encouraging since complex topologies can be obtained with a fast convergence. However, our algorithm is still unable to address some issues like the blending constraint which ensures the continuity of the stacking sequence through multiple panels.

\section{Setting of the problem}

\subsection{Multi-layer and multi-shape composite design}

Let $\mathcal{L}$ be a symmetric laminated composite structure composed of the superposition of $2 N$ anisotropic layers, each one of constant thickness $\varepsilon>0$ and characterized by a shape $\Omega_{i} \subset \Omega$, where $\Omega$ is a regular sub-domain of $\mathbb{R}^{2}$ (typically a rectangle). This symmetry assumption is made for the sake of simplicity but it is not crucial for the sequel. Note that, in some cases, non-symmetric designs may perform better [79]. We denote by $\mathcal{O}$ the collection of shapes

$$
\mathcal{O}=\left(\Omega_{i}\right)_{i=-N, \ldots,-1,+1, \ldots,+N} .
$$

Since we suppose $\mathcal{L}$ symmetric, i.e., $\Omega_{-i}=\Omega_{i}$, we consider only $N$ layers, so from now on we rather write $\mathcal{O}=\left(\Omega_{i}\right)_{i=1, \ldots . N}$. The index $i$ grows from the inside to the outside of the laminated composite structure (see Figure 1).

Each layer is made of an orthotropic material, i.e., an anisotropic material where there are three mutually perpendicular planes of symmetry in material properties. In the case of an unidirectional reinforced composite, the material properties, which are that of an equivalent homogeneous orthotropic continuum, are thus parametrized by an angle of rotation, corresponding to the orientation (at the microscopic level) of the fibers with respect to the canonical axis.

For the purpose of this study, the shape of each layer $\Omega_{i}$ and their superposition within the multilayered structure can be understood in two different ways (see Figure 2).

- Configuration I: Each layer is a non-homogeneous two-phase material, where each "hole" is filled with another "weak" material with different physical properties (weight, electric or heat conductivity, etc.). We will denote this weak material as $\mathcal{A}_{0}$. We will mainly focus on this configuration.

- Configuration II: The holes in each ply are really "void" (in this case $\mathcal{A}_{0}=0$ ) and, when gluing together the plies towards the plane of symmetry $\Pi=0$ (inwardly blended), the distal layer bends and fills the holes to keep contact with the proximal layers. The mostly outer layers will be always full in order to avoid the existence of holes throughout the laminate.

Let $\chi_{i}$ be the characteristic function of the $i$-layer. According to the classical laminate theory for plates [23, 64, the composite structure $\mathcal{L}$ is characterized by the superposition of the elastic properties 


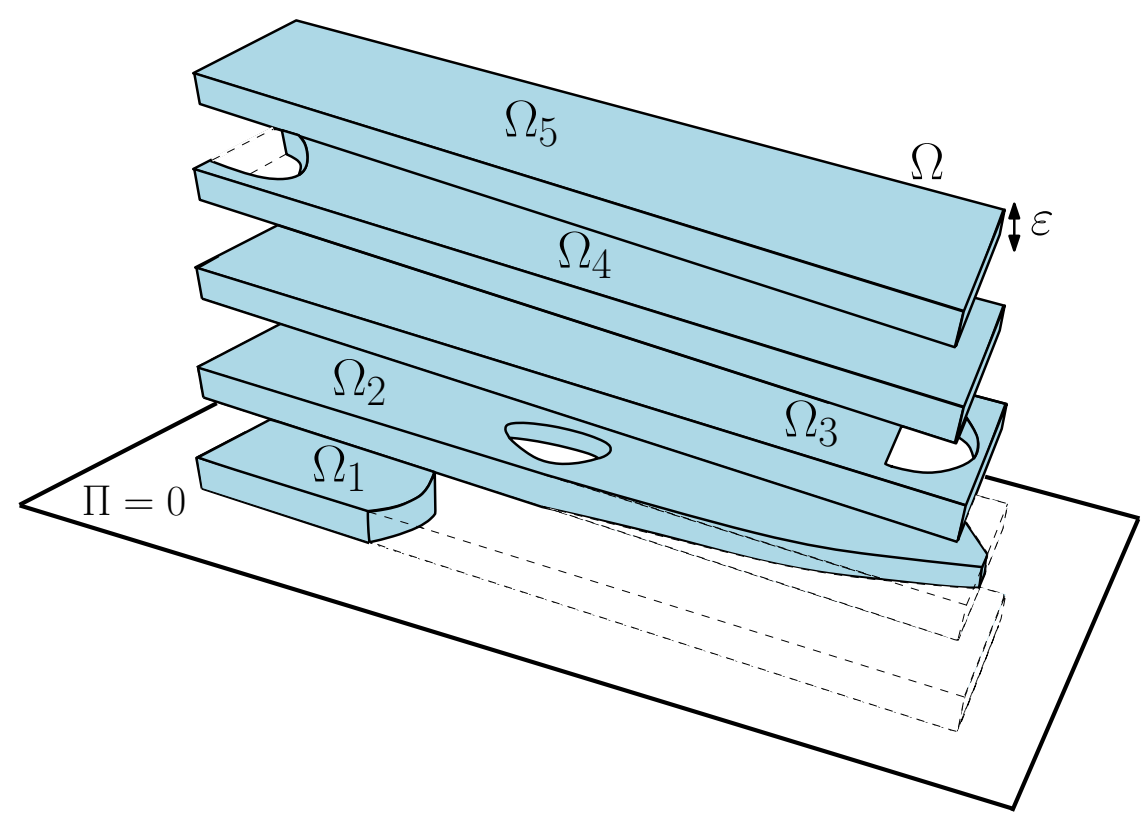

Figure 1: Half-part of a multi-shape composite design with plane of symmetry $\Pi=0$. Each ply has its own shape $\Omega_{i} \subset \Omega$.

of each layer, namely the extensional stiffness tensor $\mathcal{A}$, which reads

$$
\mathcal{A}(x)=2 \varepsilon \sum_{i=1}^{N}\left(\chi_{i}(x) \mathcal{A}_{i}+\left(1-\chi_{i}(x)\right) \mathcal{A}_{0}\right)
$$

where $\mathcal{A}_{i}$ is the extensional stiffness of the $i$-layer (a symmetric fourth-order tensor), and the bending stiffness tensor $\mathcal{D}$, which reads

$$
\mathcal{D}(x)=\frac{2 \varepsilon^{3}}{3} \sum_{i=1}^{N}\left\{\left(i^{3}-(i-1)^{3}\right)\left(\chi_{i} \mathcal{A}_{i}+\left(1-\chi_{i}\right) \mathcal{A}_{0}\right)\right\}
$$

for configuration I meanwhile for configuration II

$$
\mathcal{D}(x)=\frac{2 \varepsilon^{3}}{3} \sum_{i=1}^{N}\left\{\left(\left(\sum_{j=1}^{i} \chi_{j}(x)\right)^{3}-\left(\sum_{j=1}^{i-1} \chi_{j}(x)\right)^{3}\right) \mathcal{A}_{i}\right\} .
$$

The factor "2" in the definition of the above tensors is due to the symmetry of the laminate. The boundary of $\Omega$ is decomposed into two disjoints subsets $\partial \Omega=\Gamma_{N} \cup \Gamma_{D}, \Gamma_{N} \cap \Gamma_{D}=\emptyset$. On $\Gamma_{N}$ a in-plane surface load is applied, $g \in L^{2}\left(\Gamma_{N} ; \mathbb{R}^{2}\right)$, and on $\Gamma_{D}$ the in-plane and vertical displacements are fixed to zero. Define the spaces

$$
\begin{aligned}
& H_{D}^{1}\left(\Omega ; \mathbb{R}^{2}\right)=\left\{v \in H^{1}\left(\Omega ; \mathbb{R}^{2}\right) \text { such that } v=0 \text { on } \Gamma_{D}\right\} \\
& H_{D}^{2}(\Omega)=\left\{\eta \in H^{2}(\Omega) \text { such that } \eta=\nabla \eta \cdot n=0 \text { on } \Gamma_{D}\right\}
\end{aligned}
$$

Our mechanical model is the linearized buckling problem for the two-dimensional von Kármán plate model [19, 64]. The unknowns are the in-plane displacement $u \in H_{D}^{1}\left(\Omega ; \mathbb{R}^{2}\right)$, the vertical displacement $w \in H_{D}^{2}(\Omega), w \neq 0$, and the so-called "buckling load factor" or "buckling critical reserve factor" $\lambda \in \mathbb{R}$. They satisfy

$$
\left\{\begin{array}{cc}
\nabla^{2}:\left(\mathcal{D} \nabla^{2} w\right)=\lambda(\mathcal{A} e(u)): \nabla^{2} w & \text { in } \Omega, \\
w=0, \nabla w \cdot n=0 & \text { on } \Gamma_{D}, \\
\left(\mathcal{D} \nabla^{2} w\right)_{n n}=0 & \text { on } \Gamma_{N}, \\
\nabla \cdot\left(\mathcal{D} \nabla^{2} w\right) \cdot n+\frac{\partial}{\partial \tau}\left(\mathcal{D} \nabla^{2} w\right)_{n \tau}=\lambda 2 N \varepsilon g \cdot \nabla w & \text { on } \Gamma_{N},
\end{array}\right.
$$




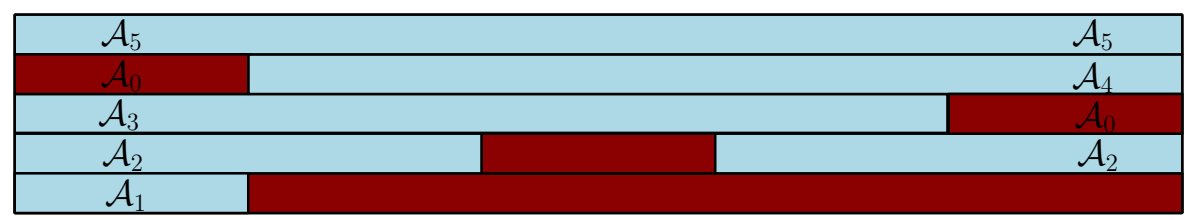

(a) Configuration I. Multi-material.

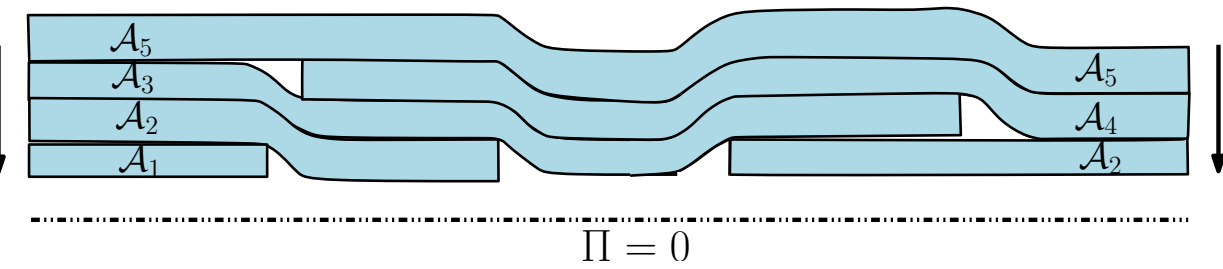

(b) Configuration II. Inwardly blended.

Figure 2: Example of transversal cut of a multi-shape composite. For simplification the cut was divided into 5 panels. Different stacking sequence are defined according to each configuration.

and

$$
\left\{\begin{array}{cc}
-\operatorname{div}(\mathcal{A} e(u))=0 & \text { in } \Omega \\
u=0 & \text { on } \Gamma_{D} \\
\mathcal{A} e(u) \cdot n=2 N \varepsilon g, & \text { on } \Gamma_{N}
\end{array}\right.
$$

where $e(u)=\frac{\nabla u+(\nabla u)^{T}}{2}$ is the classical linearized strain tensor, $(n, \tau)$ is the orthonormal local basis of normal and tangent vectors on $\partial \Omega$.

One first solves the linearized elasticty equation (5), then one solves the spectral problem (4) where $\lambda$ is an eigenvalue and $w$ an eigenfunction (while $u$ plays the role of a coefficient in the right hand side). The last boundary condition in (4) stands for the free vertical displacement on $\Gamma_{N}$ and represents the equilibrium between the bending moments and the shear forces [15, 69. We denote by $\lambda_{1}$ the smallest positive eigenvalue of (4). This is the only eigenvalue with a physical meaning: it is the buckling load factor which is an indicator of the degree of safety against buckling [31. The computed vertical displacement eigenfunction $w_{1}$ is referred here as the "buckling mode".

Throughout this paper we use Einstein's summation convention. The colon stands for the double contraction as in

$$
\mathcal{A} e(u): \nabla^{2} w=\mathcal{A}_{i j k l} e(u)_{k l} \partial_{i j}^{2} w, \quad \nabla^{2}:\left(\mathcal{D} \nabla^{2} w\right)=\partial_{i j}^{2}\left(\mathcal{D}_{i j k l} \partial_{k l}^{2} w\right),
$$

where $\mathcal{A}, \mathcal{D}$ are fourth-order tensors, $\partial_{i}$ and $\partial_{i j}^{2} w$ are the first and second-order partial derivatives, respectively, of $w$ with respect to $x_{i}$ and $x_{j}$, and $e(u)_{i j}=\left(\partial_{i} u_{j}+\partial_{j} u_{i}\right) / 2$. Thus the variational formulation of the linearized buckling problem (4)-(5) reads

$$
\begin{aligned}
\int_{\Omega} \mathcal{D}_{i j k l} \partial_{k l}^{2} w \partial_{i j}^{2} \eta d x & =-\lambda \int_{\Omega} \mathcal{A}_{i j k l} e(u)_{k l} \partial_{i} w \partial_{j} \eta d x \quad \forall \eta \in H_{D}^{2}(\Omega), \\
\int_{\Omega} \mathcal{A}_{i j k l} e(u)_{k l} e(v)_{i j} d x & =2 N \varepsilon \int_{\Gamma_{N}} g_{i} v_{i} d s \quad \forall v \in H_{D}^{1}\left(\Omega ; \mathbb{R}^{2}\right) .
\end{aligned}
$$

The existence of discrete eigenvalues $\lambda$ for (4) is guaranteed by the compactness of the buckling operator (see Lemma 2.2-7 in [20]). Furthermore this operator is self-adjoint so the smallest positive eigenvalue $\lambda_{1}$ can be express through the Rayleigh quotient

$$
\frac{1}{\lambda_{1}}=\max _{w \in H_{D}^{2}(\Omega) \backslash\{0\}} \max \left(0,-\frac{\int_{\Omega} \mathcal{A} e(u) \cdot \nabla w \cdot \nabla w d x}{\int_{\Omega} \mathcal{D} \nabla^{2} w: \nabla^{2} w d x}\right) .
$$

Note that, if there is no function $w$ such that $\int_{\Omega} \mathcal{A} e(u) \cdot \nabla w \cdot \nabla w d x \leq 0$, the right hand side in (7) is zero, which implies that there is no positive finite eigenvalue for (4). In other words, the buckling load factor is infinite.

Remark 2.1. The set of equations (4),5) describe the laminate behavior in the framework of the classical laminate theory. We could also have used more realistic laminate models, such as the first-order 
shear deformation theory or the third-order shear deformation theory (see [64] for details), which have the advantage of taking into account the influence of the transverse shear stiffness of the plate on its mechanical response. In both cases the computation of $\lambda_{1}$ (which remains a good measure of buckling risk) would have been slightly different from the one presented. For instance new terms in the numerator of the Rayleigh quotient (7) would have appeared. We chose however the classical laminate theory in order to simplify the mechanical description of the composite laminate and thus focus on the topology mixed integer character of the optimization problem.

\subsection{Stacking sequence}

Even though the fiber orientation might take any possible rotation angle, in real applications due to manufacturing constraints, it only takes discrete values [30. We will consider four values, namely: $0^{0}, 90^{0}, 45^{0},-45^{0}$. We denote by $\mathcal{C}_{0^{\circ}}, \mathcal{C}_{90^{\circ}}, \mathcal{C}_{45^{\circ}}, \mathcal{C}_{-45^{\circ}}$ their respective in-plane reduced stiffness tensors. We assume that the fiber orientation is constant in each ply.
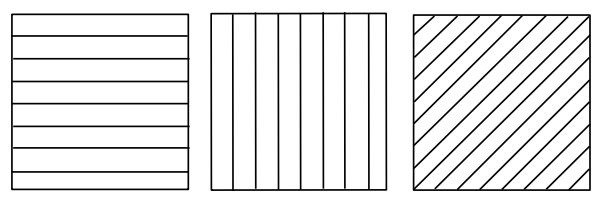

Figure 3: Fiber orientation of $0^{0}, 90^{0}, 45^{0},-45^{0}$.

Definition 2.1. We define the stacking sequence as the set of ply orientations and the way they are arranged in the normal direction of the composite laminate (see Figure 1). We represent it through a binary matrix $\xi=\left(\xi_{i j}\right) \in\{0,1\}$, where $i=1, \ldots, N, j=1,2,3,4$, and

$$
\xi_{i j}= \begin{cases}1, & \text { if the layer in position } i \text { has fiber orientation } j, \\ 0, & 1 \text { if not. }\end{cases}
$$

We identify fiber orientations $1,2,3,4$ to the angles $0^{0}, 90^{0}, 45^{0},-45^{0}$, respectively. A compatibility constraint must be imposed to obtain one and only one orientation in each ply, namely

$$
\sum_{j=1}^{4} \xi_{i j}=1, \quad \text { for any } i=1, \ldots, N
$$

Remark 2.2. We recall that, since the laminate $\mathcal{O}$ is symmetric, only half of the stacking sequence is studied and thus encoded in the matrix $\xi$.

Thanks to the above definition, the extension stiffness tensor of the laminate can be parametrized as

$$
\mathcal{A}_{i}(\xi)=\xi_{i 1} \mathcal{C}_{0^{\circ}}+\xi_{i 2} \mathcal{C}_{90^{\circ}}+\xi_{i 3} \mathcal{C}_{45^{\circ}}+\xi_{i 4} \mathcal{C}_{-45^{\circ}},
$$

and thus the stiffness tensors $\mathcal{A}(\mathcal{O}, \xi), \mathcal{D}(\mathcal{O}, \xi)$ are functions of the shapes of the laminate $\mathcal{O}$ and the stacking sequence $\xi$.

Remark 2.3. Even though by definition $\xi$ is a discrete variable, from now on, the properties of a general function depending on $\xi$ will be described by abuse of language as supposing that each component of $\xi$ was continuous in the interval $[0,1]$. In particular the tensors $\mathcal{A}$ and $\mathcal{D}$ are "linear functions" of $\xi$. This makes sense in view of the many optimization algorithms which relax the discrete character of $\xi$.

Lemma 2.1. Suppose that every ply of the laminated composite has the same shape $\Omega^{*} \subset \Omega$, namely $\Omega_{i}=\Omega^{*}$ for $i=1, \ldots, N$. Then, if the proportion of plies of each fiber orientation $j=1, \ldots, 4$ is fixed within the laminate, the tensor $\mathcal{A}$ and the in-plane displacement $u$, solution of (5), do not depend on the choice of the stacking sequence $\xi$, as long as $\xi$ satisfies these proportions.

Proof. Let $p_{j}$ with $j=1,2,3,4$, be the proportion of plies of each fiber orientation within the composite laminate. By definition, $p_{j}=\frac{1}{N} \sum_{i=1}^{N} \xi_{i j}$. Denote as $\chi^{*}$ the characteristic function of $\Omega^{*}$. Thanks to the 
linear structure of the extensional stiffness tensor $\mathcal{A}$ in equations (1) or (8), we get

$$
\begin{aligned}
\mathcal{A} & =2 \varepsilon\left(\sum_{i=1}^{N} \chi^{*} \mathcal{A}_{i}\right)+2 \varepsilon N\left(1-\chi^{*}\right) \mathcal{A}_{0}=2 \varepsilon \chi^{*}\left(\sum_{i=1}^{N} \sum_{j=1}^{4} \xi_{i j} \mathcal{C}_{j}\right)+2 \varepsilon N\left(1-\chi^{*}\right) \mathcal{A}_{0} \\
& =2 \varepsilon \chi^{*} N\left(\sum_{j=1}^{4} \mathcal{C}_{j} \frac{1}{N} \sum_{i=1}^{N} \xi_{i j}\right)+2 \varepsilon N\left(1-\chi^{*}\right) \mathcal{A}_{0}=2 \varepsilon \chi^{*} N \sum_{j=1}^{4} \mathcal{C}_{j} p_{j}+2 \varepsilon N\left(1-\chi^{*}\right) \mathcal{A}_{0} .
\end{aligned}
$$

Hence if $p_{j}$ remains constant, so does the tensor $\mathcal{A}$ and the in-plane displacement $u$, solution of (5).

Remark 2.4. Definition 2.1 of the stacking sequence is not unique: there are other possible choices. Our present choice corresponds to a permutation of the material properties in the layers, irrespective of the shape $\Omega_{i}$ which is thus attached to the $i$-th layer. Another choice could have been, on the contrary, to bind the shape $\Omega_{i}$ with the material properties of its layer $i$ and, then, to permute this couple of shape and material properties. This latter choice, however elegant, has the dramatic consequence of considerably increasing the number of components of the new variable $\xi$ (from $4 N$ to $N$ !). Furthermore, the expressions of the tensor $\mathcal{D}$ in (2) and (3) are more complex (notably non-linear with respect to $\xi$ ). Nevertheless, it would have one advantage: Lemma 2.1 would hold true without any assumption on the constant shape $\Omega_{i}=\Omega^{*}$, namely the tensor $\mathcal{A}$ and the in-plane displacement $u$ would be independent of $\xi$. In any case, the inconvenients overcome the advantages, so we stick to our former Definition 2.1.

\subsection{Goal of the present study}

We look for a multi-layered composite plate with optimal stacking sequence and optimal ply shapes. Typically the optimization problem will be set as a mass minimization problem subject to a set of manufacturing constraints, local failure criteria, in-plane stiffness and avoidance of buckling.

\subsubsection{General problem}

From a mathematical point of view, our problem can be casted as a mixed optimization problem, namely

$$
\left\{\begin{array}{l}
\min _{\mathcal{O} \in \mathcal{U}_{a d}, \xi \in \mathcal{Y}_{a d}} J(\mathcal{O}) \\
\text { such that } G(\mathcal{O}, \xi) \leq 0
\end{array}\right.
$$

Problem $(9)$ is called mixed because $\mathcal{O}$ is a continuous variable while $\xi$ is a discrete one. The objective function $J(\mathcal{O})$ is the mass of the structure

$$
J(\mathcal{O})=\rho \sum_{i=1}^{N} \int_{\Omega} \chi_{i} d x,
$$

where the material density $\rho$ is assumed to be constant and the mass of the weak material $\mathcal{A}_{0}$ is neglected. The cost function $J(\mathcal{O})$ does not depend on the stacking sequence $\xi$. The function $G:\left(\mathcal{U}_{a d} \times \mathcal{Y}_{a d}\right) \rightarrow \mathbb{R}^{m}$ is a regular vector-valued constraint function with $m$ components. Typically $G$ is a mechanical constraint on the stiffness of the plate. Notably, we focus our attention on two kinds of stiffness measures, namely

$$
G(\mathcal{O}, \xi)=\left\{\begin{array}{l}
\left(\lambda_{1}^{-1}(\mathcal{O}, \xi)-1\right) \\
\int_{\Omega} s(x, \mathcal{O}, \xi, u(\mathcal{O}, \xi), \nabla u(\mathcal{O}, \xi)) d x
\end{array}\right.
$$

The first criterion expresses the buckling avoidance through the load factor or first positive eigenvalue of (4), meanwhile the second criterion represents a global failure or stiffness criterion, with $s$ a regular function. This last functional could be e.g. the compliance or an averaged Tsai-Hill failure criterion (adaptation to composites of the von Mises criterion for isotropic or more generally cubic-symmetry materials).

Remark 2.5. Note that for the last criterion in (11), the state equation reduces to (5) (the solution of (4) is not required).

In (9) the sets $\mathcal{Y}_{a d}$ and $\mathcal{U}_{a d}$ are the sets of admissible stacking sequences and admissible shapes, respectively. An explicit definition of the set $\mathcal{Y}_{a d}$ (including manufacturing constraints) will be given in 
Section 3. We define the set of admissible shapes $\mathcal{U}_{a d}$ as the $N$-collection of open subsets of the working domain $\Omega$ with bounded perimeter

$$
\mathcal{U}_{a d}=\left\{\left\{\Omega_{i}\right\}_{i=1, \ldots, N} \text { with } \Omega_{i} \subset \Omega \text { and } \operatorname{Per}\left(\Omega_{i}\right) \leq \varsigma, \forall i=1, \ldots, N\right\},
$$

where $\operatorname{Per}(A)=\int_{\partial A} d s$ is the perimeter of the open set $A \subset \mathbb{R}^{2}$ and $\varsigma>0$ is a fixed constant (the same for all open sets in $\left.\mathcal{U}_{a d}\right)$. The existence of an optimal solution to problem $(9 \mathrm{p}$ relies essentially on the definition of $\mathcal{U}_{a d}$. A rigorous proof of the existence of an optimal shape of (9) can be found in [23], and it is based on classical ideas of [9].

Remark 2.6. For some applications, controlling the perimeter of each layer is important, even though we have no idea of a reference value for $\varsigma$. In that case the functional $J(\mathcal{O})$ can be re-defined as a linear combination of the weight and the total perimeter, which is also independent of the stacking sequence $\xi$

$$
J(\mathcal{O})=\sum_{i=1}^{N}\left\{\rho \int_{\Omega} \chi_{i} d x+\gamma \operatorname{Per}\left(\Omega_{i}\right)\right\}, \gamma>0 .
$$

\subsubsection{Simplification of the buckling problem}

In order to reduce the numerical cost of solving the eigenvalue problem (4) at each iteration, it is common in engineering practice to replace the true buckling load factor $\lambda_{1}$ by an approximation, called the "reserve factor", the evaluation of which merely relies on the elasticity equation (5). In the specific setting of a rectangular panel loaded as described in Figure 5 , the reserve factor is defined by

$$
\lambda_{R F}(\mathcal{O}, \xi):=\min _{x \in \Omega}\left(\frac{\frac{2 \pi^{2}}{b^{2}}\left(\sqrt{\mathcal{D}_{11} \mathcal{D}_{22}}+\mathcal{D}_{12}+2 \mathcal{D}_{33}\right)}{\left|(\mathcal{A} e(u))_{x x}^{-}\right|}\right) .
$$

and the first constraint in 11 is replaced by $\lambda_{R F}^{-1}(\mathcal{O}, \xi)-1 \leq 0$. The factor

$$
\frac{2 \pi^{2}}{b^{2}}\left(\sqrt{\mathcal{D}_{11} \mathcal{D}_{22}}+\mathcal{D}_{12}+2 \mathcal{D}_{33}\right)
$$

stands for the value of the buckling load of an homogeneous simply supported composite plate with large aspect ratio (length/width $\gg 1$ ) under compression ( $x$-axis) 64. The constant $b$ is the width of the plate and $(\mathcal{A} e(u))_{x x}^{-}$(which is the negative part of the $x x$ component of the stress tensor) corresponds to the projection of the negative (compressed) values of the stress tensor on the $x$-axis. We remark that the matrix

$$
\left(\begin{array}{ccc}
\mathcal{D}_{11} & \mathcal{D}_{12} & 0 \\
\mathcal{D}_{12} & \mathcal{D}_{22} & 0 \\
0 & 0 & \mathcal{D}_{33}
\end{array}\right)
$$

is defined as the Voigt notation matrix of the fourth-order tensor $\mathcal{D}$ for so-called specially orthotropic plates, i.e. plates for which the bending-stretching coupling and bending-twisting $\left(\mathcal{D}_{13}, \mathcal{D}_{23}\right)$ coefficients are zero [64]. When we replace the first line of (11) by (14) as a constraint in (9), the optimization problem is much simplified since the only required state equation is (5), alleviating the numerical cost of solving (4). Nevertheless, $\lambda_{R F}$ remains a coarse approximation of $\lambda_{1}$ in a more general setting, notably when there exists coupled compression/shear loads and the plates are non-homogeneous. Thus, a byproduct of this study is to also compare numerically the optimal solution of 9 for both choices of $G$ in terms of $\lambda_{1}$ or $\lambda_{R F}$. Our conclusion (see Subsection 5.6) is that $\lambda_{R F}$ is not a good approximation of $\lambda_{1}$ in our optimization setting.

Remark 2.7. Note that $\lambda_{R F}$ is defined by (14) as a minimal value. It is well known that the min function is not differentiable. Therefore, for numerical purposes the inverse of min, which is a max, will be approximated by a $L^{p}(\Omega)$-norm for some integer value of $p \geq 2$.

\subsection{General decomposition framework}

In the composite problem $(9)$ the optimization with respect to the stacking sequence $\xi$ is combinatorial (with a finite admissible set $\mathcal{Y}_{a d}$ ) while that with respect to the shapes $\mathcal{O}$ is continuous (with an infinite dimensional admissible set $\mathcal{U}_{a d}$. Therefore, both optimizations require very different techniques and it 
makes sense to work in the framework of decomposition methods 31 that we now introduce. Decomposition techniques are used to solve large-scale (or complex) problems, replacing them by a sequence of reduced or easier local problems called followers linked by a leader or master program. This gives rise to a bi-level optimization scheme 24. These methods prove efficient when the structure of the problem is naturally separable. This separability comes for instance from the structure of the objective function and constraints (e.g. data is block-separable) or when the variables can be split into "complicated" and "simple" ones, for example. We present notably an infinite dimensional adaptation of the scheme Maxmargin (from maximum margin or constraint margin) [12, 33.

Problem (9) turns out to be a quasi separable subsystem [33. Thanks to this particular structure, (9) can be writen as a bi-level optimization problem whose higher level only relies on the variable $\mathcal{O}$ through a Maxmargin scheme, as follows.

Proposition 2.1. A collection of shapes $\mathcal{O}^{*} \in \mathcal{U}_{a d}$ is a global minimizer of (9) if and only if it is a global minimizer of

$$
\min _{\mathcal{O} \in \mathcal{U}_{a d}}\{J(\mathcal{O}) \mid \mathcal{M}(\mathcal{O}) \leq 0\}
$$

where the constraint margin function $\mathcal{M}$ reads

$$
\mathcal{M}(\mathcal{O}):=\min _{\xi \in \mathcal{Y}_{a d}} \max _{1 \leq i \leq m} G_{i}(\mathcal{O}, \xi)
$$

Proof. Define the set

$$
V=\left\{\mathcal{O} \in \mathcal{U}_{a d} \mid \exists \xi \in \mathcal{Y}_{a d} \text { such that } G(\mathcal{O}, \xi) \leq 0\right\}
$$

It is straightforward that $\mathcal{O}^{*}$ solves $(9)$ if and only if it solves $\min \{J(\mathcal{O}) \mid \mathcal{O} \in V\}$. On the other hand, $\mathcal{O} \in V$ if and only if

$$
\mathcal{M}(\mathcal{O})=\min _{\xi \in \mathcal{Y}} \max _{1 \leq i \leq m}\left\{G_{i}(\mathcal{O}, \xi)\right\} \leq 0
$$

Therefore 16 is equivalent to $(9)$.

Problem (16) is called the master problem while $(17)$ is the follower problem. Problem 16 has the advantage with respect to $(9)$ to optimize only over $\mathcal{U}_{a d}$ and not over $\mathcal{U}_{a d} \times \mathcal{Y}_{a d}$. The local and global characters of the minimizers of (16) are analysed in [23. Generally the constraint margin function $\mathcal{M}$ is non-smooth and is not known analytically. As a consequence the bi-level problem (16) is usually non-differentiable. In order to write the first order optimality conditions (stationarity conditions) of problem (16), the notion of generalized gradient is needed. For a developpement of these conditions and an adapted version of a feasible direction method to solve $[16)$, consult [23].

\section{Stacking sequence optimization}

\subsection{Manufacturing constraints}

From an engineering point of view, when a composite laminate is designed, some additional composite design rules must be respected. Following the typical industrial approach (see e.g. the examples from Airbus in [12, 17]) we consider the following rules

- (R1) Continuity rule, no more than 4 successive plies with the same angle.

- (R2) Disorientation rule, maximum gap between two adjacent (superposed) plies is $45^{\circ}$.

- (R3) Balanced laminate with respect to the principal direction $0^{\circ}$, i.e. same number of plies at $45^{\circ}$ and $-45^{\circ}$.

- (R4) Minimum proportion of each fiber orientation (typically $8 \%$ ). We note this proportion as $p_{j}$, $j=1,2,3,4$.

- (R5) Symmetric laminate. This ensures to avoid the coupling between in-plane traction and bending of the plate. 
The rule (R5) is satisfied by just studying half of the stacking sequence. The rules (R1) to (R4) have no deep physical meaning and are just typical manufacturing constraints which may become obsolete in case of further progresses in the manufacturing of laminated composites. The last rule (R5) is quite restrictive since it is only a sufficient (but not necessary) condition for avoiding the coupling between in-plane traction and bending of the plate. Clearly those rules restrict the design space and dramatically better designs could be obtained if they were not taken into account. Thanks to our Definition 2.1 of the stacking sequence matrix $\xi$, all the above manufacturing constraints can easily be formulated as linear inequalities or equalities.

Proposition 3.1. Let $\{0,1\}^{N \times 4}$ be the set of binary matrices of $N$ rows and 4 columns. Define the applications $r_{1}:\{0,1\}^{N \times 4} \rightarrow \mathbb{R}^{4(N-8)}, r_{2}:\{0,1\}^{N \times 4} \rightarrow \mathbb{R}^{2(N-1)}, r_{3}:\{0,1\}^{N \times 4} \rightarrow \mathbb{R}, r_{4}:\{0,1\}^{N \times 4} \rightarrow$ $\mathbb{R}^{4}$. Then the design rules (R1), (R2), (R3), (R4) can be expressed as linear constraints with respect to the stacking sequence matrix $\xi$ as follows

$$
(R 1) \Leftrightarrow r_{1}(\xi)= \begin{cases}\sum_{i=k}^{k+3} \xi_{i j}-4 \leq 0 & \forall j=1,2,3,4, \forall k=1, \ldots, N-3, \\ 2 \xi_{1 j}+\sum_{i=2}^{3} \xi_{i j}-4 \leq 0 & \forall j=1,2,3,4, \\ \sum_{i=1}^{2} \xi_{i j}-2 \leq 0 & \forall j=1,2,3,4,\end{cases}
$$

because of the plane of symmetry,

$$
\begin{gathered}
(R 2) \Leftrightarrow r_{2}(\xi)= \begin{cases}\xi_{i 1}+\xi_{(i+1) 2}-1 \leq 0 \quad \forall i=1, \ldots, N-1 . \\
\xi_{i 3}+\xi_{(i+1) 4}-1 \leq 0 \quad \forall i=1, \ldots, N-1 .\end{cases} \\
(R 3) \Leftrightarrow \quad r_{3}(\xi)=\sum_{i=1}^{N}\left(\xi_{i 3}-\xi_{i 4}\right)=0 . \\
(R 4) \Leftrightarrow \quad r_{4}(\xi)=p_{j} N-\sum_{i=1}^{N} \xi_{i j} \leq 0, \quad \forall j=1, \ldots, 4 .
\end{gathered}
$$

Remark 3.1. The above constraint functions $r_{1}(\xi), r_{2}(\xi), r_{3}(\xi), r_{4}(\xi)$ are affine hence convex. In particular, the equality constraint $r_{3}(\xi)=0$ can be replaced by two inequalities $r_{3}(\xi) \leq 0$ and $r_{3}(\xi) \geq 0$, both of them being affine, thus convex.

Once the design rules have been established, the definition of the feasible stacking sequence set $\mathcal{Y}_{a d}$ follows.

Definition 3.1. We define the feasible stacking sequence set $\mathcal{Y}_{a d}$ as

$$
\mathcal{Y}_{a d}=\left\{\xi=\left(\xi_{i j}\right) \in\{0,1\}^{N \times 4} \text { such that } \sum_{j=1}^{4} \xi_{i j}=1, \forall i ; r_{1}(\xi) \leq 0 ; r_{2}(\xi) \leq 0 ; r_{3}(\xi)=0 ; r_{4}(\xi) \leq 0\right\}
$$

Remark 3.2. The definition of $\mathcal{Y}_{a d}$ is independent of the shapes $\mathcal{O}$ (cf. Remark 2.4). This simplification implies that the manufacturing rules are global but independent of the shape of each ply.

\subsection{Convexity properties of the constraint margin function}

In order to solve problem (9) with a decomposition technique (as explained in Subsection 2.4), one has to evaluate the constraint margin function $\mathcal{M}(\mathcal{O})$ introduced in (17). Its scalar version is defined as

$$
\mathcal{M}(\mathcal{O})=\min _{\xi \in \mathcal{Y}_{a d}} G(\mathcal{O}, \xi)
$$

where $G(\mathcal{O}, \xi)$ represents a regular measure of the stiffness of the plate. In other words, the constraint margin function represents the value of maximum slackness of the constraint $G \leq 0$. The evaluation 
of $\mathcal{M}(\mathcal{O})$ implies solving a non-linear integer problem. In general, this kind of problems are quite hard to solve due to the discrete nature of the variables. However, when the problem is linear, quadratic or convex, there exists a collection of algorithms which can be quite efficient [43. In what follows, we study the convexity of $G$ when it is either the compliance, or $\lambda_{1}^{-1}$ or $\lambda_{R F}^{-1}$.

Proposition 3.2. Let $C(\mathcal{O}, \xi)$ be the in-plane compliance of the composite plate

$$
C(\mathcal{O}, \xi)=\frac{1}{2} \int_{\Omega} \mathcal{A}(\mathcal{O}, \xi) e(u(\mathcal{O}, \xi)): e(u(\mathcal{O}, \xi)) d x,
$$

with $u(\mathcal{O}, \xi)$ solution of (5). Then for a fixed $\mathcal{O}$, the in-plane compliance function $C$ is convex with respect to $\xi$.

Proof. According to its definition, $C$ can be written as the opposite of the minimum of the elastic energy functional, namely

$$
C(\mathcal{O}, \xi)=-\min _{v \in H_{D}^{1}(\Omega)}\left(\frac{1}{2} \int_{\Omega} \mathcal{A}(\mathcal{O}, \xi) e(v): e(v) d x-2 N \varepsilon \int_{\Gamma_{N}} g \cdot v d s\right) .
$$

The desired result is deduced from the affine (thus concave) character of $\mathcal{A}$ with respect to $\xi$, the fact that the minimum of a set of concave functions is concave, and the minus sign in front of 20) which transforms concavity into convexity.

Proposition 3.3. Suppose that every ply of the laminate has the same shape $\Omega^{*} \subset \Omega$, namely $\Omega_{i}=\Omega^{*}$ for $i=1, \ldots, N$. Then, if the proportions of plies of each fiber orientation is fixed in the laminate, for every $\xi \in \mathcal{Y}_{\text {ad }}$ respecting these proportions, the functions $\lambda_{1}^{-1}(\mathcal{O}, \xi)$ and $\lambda_{R F}^{-1}(\mathcal{O}, \xi)$ are convex with respect to $\xi$.

Proof. Define the function

$$
l(\xi, w)=-\frac{\int_{\Omega} \mathcal{A}(\xi) e(u(\xi)) \cdot \nabla w \cdot \nabla w d x}{\int_{\Omega} \mathcal{D}(\xi) \nabla^{2} w: \nabla^{2} w d x}, \xi \in \mathcal{Y}_{a d}, w \in H_{D}^{2}(\Omega),
$$

and denote as $\overline{\mathcal{Y}_{a d}}$ the subset of $\xi \in \mathcal{Y}_{a d}$ respecting the fiber orientation proportions. If we fix a vertical displacement $w$, then, thanks to Lemma 2.1, for every $\xi \in \overline{\mathcal{Y}_{a d}}$ the numerator of 21) is constant (in particular it does not change sign), meanwhile the denominator is a positive linear function of $\xi$. Furthermore let $\Lambda \subset H_{D}^{2}(\Omega)$ be the set of functions $w$ such that $l(\xi, w)>0 \forall \xi \in \overline{\mathcal{Y}_{a d}}$. Since we suppose the existence of $\lambda_{1}>0$, then $\Lambda \neq \emptyset$. Hence, applying Lemma 3.2 for every fixed $w \in \Lambda$ the function $\xi \rightarrow l(\xi, w)$ is convex. Finally noticing that

$$
\lambda_{1}^{-1}(\xi)=\max _{w \in \Lambda} l(\xi, w),
$$

the convexity of $\lambda_{1}^{-1}(\xi)$ stems from the fact that the maximum or supremum of a set of convex functions is convex. On the other hand, by virtue of Lemma 3.1, the function 22 is concave and positive with respect to $\xi$ so, from definition (14,

$$
\lambda_{R F}^{-1}(\xi)=\max _{x \in \Omega}\left(\frac{\left|(\mathcal{A} e(u))_{x x}^{-}\right|(\xi)}{\frac{2 \pi}{b}\left(\sqrt{\mathcal{D}_{11}(\xi) \mathcal{D}_{22}(\xi)}+\mathcal{D}_{12}(\xi)+2 \mathcal{D}_{33}(\xi)\right)}\right)
$$

is equally convex for $\xi \in \overline{\mathcal{Y}_{a d}}$ in view of Lemma 3.2

Lemma 3.1. For a fixed $\mathcal{O}$, denote the numerator of $\lambda_{R F}$ in 14) as

$$
D_{R F}(\xi):=\sqrt{\mathcal{D}_{11}(\xi) \mathcal{D}_{22}(\xi)}+\mathcal{D}_{12}(\xi)+2 \mathcal{D}_{33}(\xi) .
$$

Then $D_{R F}(\xi)$ is a concave function of $\xi$.

Proof. The ply shapes $\mathcal{O}$ are fixed: therefore, although the tensor $\mathcal{D}$ depends on $\mathcal{O}$ too, we write explicitly only the dependence with respect to $\xi$. Now, since the tensor $\mathcal{D}(\xi)$ is a linear function of $\xi$, the concavity of $D_{R F}(\xi)$ reduces to prove the concavity of the term $\hat{D}(\xi):=\sqrt{\mathcal{D}_{11}(\xi) \mathcal{D}_{22}(\xi)}$. This last property is easily deduced from the concavity of $\hat{d}\left(x_{1}, x_{2}\right)=\sqrt{x_{1} x_{2}}$ in $\left(\mathbb{R}^{+}\right)^{2}$ (just compute its Hessian which is negative semidefinite).

Lemma 3.2. Let $f(\xi):=\mathcal{Y}_{a d} \rightarrow \mathbb{R}_{*}^{+}$be a twice differentiable function and define the function $g(\xi)=$ $1 / f(\xi)$. If $f(\xi)$ is positive and concave, then $g(\xi)$ is convex. In particular if $f(\xi)$ is an affine application, then $g(\xi)$ is convex if and only if $f(\xi)$ is positive.

The proof is a simple computation that we leave to the reader. 


\subsection{An algorithm for the evaluation of the constraint margin function}

Among the algorithms for discrete convex optimization, the outer approximation method (OA) is one of the most relevant [43, 62. First introduced by 25] and then extended in [27, 81, for convex problems, the algorithm solves mixed integer programming problems through a finite sequence of alternatively non linear programming subproblems (in which the integer variables are fixed) and mixed integer linear problems (MILP). The optimal solution of each subproblem provides a point at which supporting hyperplanes of the functions are generated. These linearizations are collected in an MILP master program who determines the new integer assignment for the next iteration. Even though the OA method is capable of tackling mixed integer problems, in our case we use it in the full discrete case (integer nonlinear programming).

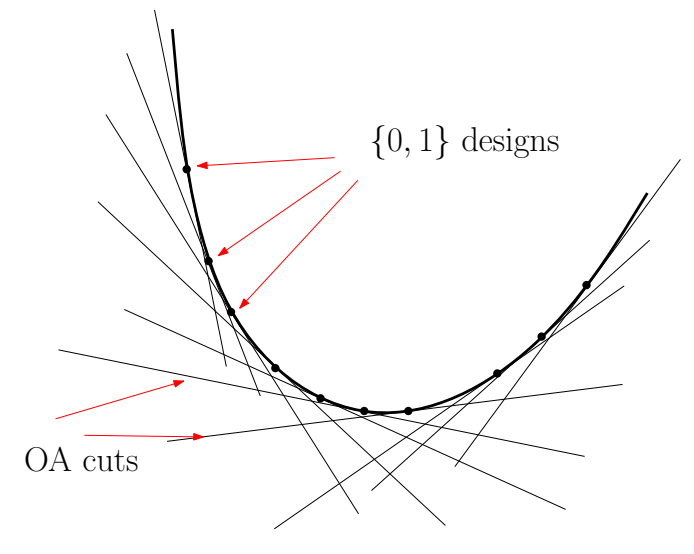

Figure 4: Scheme of the Outer Approximation (OA) method.

For $\mathcal{Y}=\{0,1\}^{N \times 4}$ and a fixed shape $\mathcal{O}$, consider problem 18 , namely

$$
\min _{\xi \in \mathcal{Y}}\{G(\xi) \mid r(\xi) \leq 0\}
$$

where $G$ is a function defined on $[0,1]^{N \times 4}$, convex, continuously differentiable and $r$ is linear. This is a valuable framework since, according to Definition 3.1 , the constraints defining $\mathcal{Y}_{a d} \subset \mathcal{Y}$ (represented by " $r \leq 0$ " in (23)) are linear and moreover, as proved in Propositions 3.2 and 3.3 , the different choices, $G=C, G=\lambda_{1}^{-1}$ or $G=\lambda_{R F}^{-1}$, are convex under certain conditions. that

Due to the convexity of $G$, for each $\hat{\xi} \in \mathcal{Y}$, its tangent hyperplane is a linear under-estimator such

$$
G(\hat{\xi})+\nabla G(\hat{\xi}) \cdot(\xi-\hat{\xi}) \leq G(\xi), \quad \forall \xi \in \mathcal{Y}
$$

Let $\epsilon>0$ be a small parameter corresponding to the required decrease at each iteration. The OA method is defined by the following algorithm.

Algorithm 3.1. Let $\xi^{0}$ be an initial feasible solution of (23), $T \subset \mathcal{Y}$ the set of generated admissible solutions so far and $\xi^{*}$ the current best computed solution. Initialize $T=\left\{\xi^{0}\right\}$ and set the upper bound constant $\mathrm{UBD}=+\infty$.

1) If $G\left(\xi^{k}\right) \leq \mathrm{UBD}-\epsilon$, then update $\xi^{*}=\xi^{k}$ and $\mathrm{UBD}=G\left(\xi^{k}\right)$.

Otherwise, do nothing.

2) Solve the current master problem:

(M) $\min _{\xi \in \mathcal{Y}, \eta \in \mathbb{R}}\{\eta \mid \eta \leq \mathrm{UBD}-\epsilon ; r(\xi) \leq 0 ; G(\hat{\xi})+\nabla G(\hat{\xi}) \cdot(\xi-\hat{\xi}) \leq \eta \forall \hat{\xi} \in T\}$.

3) If the problem is infeasible: STOP. The optimal solution is $\xi^{*}$.

4) Otherwise let $\xi^{k+1}$ be the optimal solution of (M). Update $T$ as $T=T \cup\left\{\xi^{k+1}\right\}$ and return to 1 ). 
It was proved in [27, that for $\epsilon$ small enough, the sequence of points $\left(\xi^{k}\right)_{k} \in \mathcal{Y}$ generated by the OA algorithm terminates in a finite number of steps at an optimal solution of (23).

Remark 3.3. In many situations the function $G$ is not necessarily convex (it happens, for example, for the inverse buckling load when the proportions of plies with a given fiber orientation are not fixed). Hence the convergence of the OA method to the optimum of (23) is not guaranteed. In such a case, the OA algorithm can still be used as an heuristic which furnishes a decreasing sequence of stacking configurations $\xi$. Note that it is possible to add a large term of the type $\xi(1-\xi)$ to the function $G$, which makes it convex and does not change problem [23) (see [23]). We do not use this modification of the OA algorithm in the sequel.

We end this section by giving the derivatives (with respect to $\xi$ ) of the function $G$ when it is either the compliance $C(\mathcal{O}, \xi)$ or $\lambda_{1}^{-1}(\mathcal{O}, \xi)$. These derivatives are a required ingredient in the linear underestimators of Algorithm 3.1. Since the shapes $\mathcal{O}$ are held fixed, to simplify the notation, we omit the dependence on $\mathcal{O}$ in the sequel.

Proposition 3.4. Let $C(\xi)$ be the compliance functional defined by (19). Then $C(\xi)$ is differentiable with respect to $\xi$ and the derivative reads

$$
\nabla_{\xi} C(\xi)=-\frac{1}{2} \int_{\Omega} \nabla_{\xi} \mathcal{A}(\xi) e(u(\xi)): e(u(\xi)) d x \quad \text { with } \quad \nabla_{\xi} \mathcal{A}(\xi)=\frac{\partial \mathcal{A}}{\partial \xi_{i j}}(\xi)=2 \varepsilon \chi_{i}(x) \mathcal{C}_{j} .
$$

Proof. Applying the chain rule lemma

$$
\nabla_{\xi} C(\xi)=\frac{1}{2} \int_{\Omega} \nabla_{\xi} \mathcal{A}(\xi) e(u(\xi)): e(u(\xi)) d x+\int_{\Omega} \mathcal{A}(\xi) e\left(\nabla_{\xi} u(\xi)\right): e(u(\xi)) d x,
$$

where $\nabla_{\xi} \mathcal{A}(\xi)$ is the derivative of (1). Taking the derivative in $\xi$ on both sides of (6) yields the state equation solved by $\nabla_{\xi} u(\xi)$

$$
\int_{\Omega} \mathcal{A}(\xi) e\left(\nabla_{\xi} u(\xi)\right): e(v) d x=-\int_{\Omega} \nabla_{\xi} \mathcal{A}(\xi) e(u(\xi)): e(v) d x, \quad \forall v \in H_{D}^{1}(\Omega) .
$$

The desired result follows from taking $v=u(\xi)$ and injecting (25) in 24).

Proposition 3.5. Suppose $\lambda_{1}(\xi)$ is a simple eigenvalue and normalize the corresponding eigenvector $w$ by

$$
\int_{\Omega} \mathcal{A} e(u) \cdot \nabla w \cdot \nabla w d x=-1
$$

Define the adjoint state $p \in H_{D}^{1}(\Omega)$ as the solution of

$$
\int_{\Omega} \mathcal{A} e(p): e(v) d x=\lambda_{1} \int_{\Omega} \mathcal{A} e(v) \cdot \nabla w \cdot \nabla w d x, \quad \forall v \in H_{D}^{1}(\Omega) .
$$

Then, the map $\xi \rightarrow \lambda_{1}(\xi)$ is differentiable and its derivative reads

$$
\nabla_{\xi} \lambda_{1}(\xi)=\int_{\Omega} \nabla_{\xi} \mathcal{D}(\xi) \nabla^{2} w: \nabla^{2} w d x+\lambda_{1} \int_{\Omega} \nabla_{\xi} \mathcal{A}(\xi) e(u) \cdot \nabla w \cdot \nabla w d x-\int_{\Omega} \nabla_{\xi} \mathcal{A}(\xi) e(p): e(u) d x,
$$

with

or

$$
\nabla_{\xi} \mathcal{D}(\xi)=\frac{\partial \mathcal{D}}{\partial \xi_{i j}}(\xi)=\frac{2}{3} \varepsilon^{3}\left(i^{3}-(i-1)^{3}\right) \chi_{i}(x) \mathcal{C}_{j}
$$

$$
\nabla_{\xi} \mathcal{D}(\xi)=\frac{\partial \mathcal{D}}{\partial \xi_{i j}}(\xi)=\frac{2}{3} \varepsilon^{3}\left(\left(\sum_{k=1}^{i} \chi_{k}(x)\right)^{3}-\left(\sum_{k=1}^{i-1} \chi_{k}(x)\right)^{3}\right) \mathcal{C}_{j}
$$

depending on the composite configuration (see equations (2) and (3)). 
Proof. Since it is simple, $\lambda_{1}$ and its normalized eigenvector are differentiable [65. Thus, differentiating in $\xi$ on both sides of the Rayleigh quotient (7) yields

$$
\begin{aligned}
\nabla_{\xi}\left(\lambda_{1}^{-1}\right)(\xi) & =\frac{-1}{\int_{\Omega} \mathcal{D} \nabla^{2} w: \nabla^{2} w d x}\left(\int_{\Omega}\left(\nabla_{\xi} \mathcal{A}(\xi) e(u)+\mathcal{A} e\left(\nabla_{\xi} u(\xi)\right)\right) \cdot \nabla w \cdot \nabla w d x+2 \int_{\Omega} \mathcal{A} e(u) \cdot \nabla \nabla_{\xi} w(\xi) \cdot \nabla w d x\right) \\
& -\frac{\lambda_{1}^{-1}}{\int_{\Omega} \mathcal{D} \nabla^{2} w: \nabla^{2} w d x}\left(\int_{\Omega} \nabla_{\xi} \mathcal{D}(\xi) \nabla^{2} w: \nabla^{2} w d x+2 \int_{\Omega} \mathcal{D} \nabla^{2} w: \nabla^{2} \nabla_{\xi} w(\xi) d x\right)
\end{aligned}
$$

Since $w$ satisfies $(6)$ for every $\eta \in H_{D}^{2}(\Omega)$, in particular taking $\eta=\nabla_{\xi} w(\xi)$, the above equation simplifies to

$$
\nabla_{\xi}\left(\lambda_{1}^{-1}\right)=-\lambda_{1}^{-2}\left(\lambda_{1} \int_{\Omega}\left(\nabla_{\xi} \mathcal{A}(\xi) e(u)+\mathcal{A} e\left(\nabla_{\xi} u(\xi)\right)\right) \cdot \nabla w \cdot \nabla w d x+\int_{\Omega} \nabla_{\xi} \mathcal{D}(\xi): \nabla^{2} w: \nabla^{2} w d x\right)
$$

where thanks to 26 , we have used the fact that

$$
\int_{\Omega} \mathcal{D} \nabla^{2} w: \nabla^{2} w d x=\lambda_{1}
$$

Now, it is a classical computation to eliminate $\nabla_{\xi} u(\xi)$ by using the adjoint $p$ and we deduce the desired result by using the relation $\nabla_{\xi}\left(\lambda_{1}^{-1}\right)=-\nabla_{\xi} \lambda_{1}(\xi) / \lambda_{1}^{2}(\xi)$.

\section{Shape optimization}

\subsection{Shape sensitivity analysis}

In this section we briefly recall the definition and main results about shape derivation in the context of composite optimization in dimension $d=2$. Shape differentiation is a classical topic that goes back to Hadamard [6, 36, 69]. Let the overall domain $\Omega \subset \mathbb{R}^{2}$ be fixed and bounded. Let $\Omega_{1} \subset \Omega$ be a smooth open subset which is variable. Indeed, we consider variations of the type

$$
(I d+\theta)\left(\Omega_{1}\right):=\left\{x+\theta(x) \text { for } x \in \Omega_{1}\right\}
$$

with $\theta \in W^{1, \infty}\left(\Omega ; \mathbb{R}^{2}\right)$ such that $\|\theta\|_{W^{1, \infty}\left(\Omega ; \mathbb{R}^{2}\right)}<1$ and tangential on $\partial \Omega$ (i.e., $\theta \cdot n=0$ on $\partial \Omega$; this last condition ensures that $\Omega=(I d+\theta) \Omega)$. It is well known that, for sufficiently small $\theta,(I d+\theta)$ is a diffeomorphism in $\Omega$.

Definition 4.1. The shape derivative of a function $J\left(\Omega_{1}\right)$ is defined as the Fréchet derivative in $W^{1, \infty}\left(\Omega ; \mathbb{R}^{2}\right)$ at 0 of the application $\theta \rightarrow J\left((I d+\theta) \Omega_{1}\right)$, i.e.

$$
J\left((I d+\theta) \Omega_{1}\right)=J\left(\Omega_{1}\right)+J^{\prime}\left(\Omega_{1}\right)(\theta)+o(\theta) \quad \text { with } \quad \lim _{\theta \rightarrow 0} \frac{|o(\theta)|}{\|\theta\|_{W^{1, \infty}}}=0
$$

where $J^{\prime}\left(\Omega_{1}\right)$ is a continuous linear form on $W^{1, \infty}\left(\Omega ; \mathbb{R}^{2}\right)$.

Remark 4.1. A simple example of shape derivative is, for a given function $f \in H^{1}(\Omega)$,

$$
J\left(\Omega_{1}\right)=\int_{\Omega_{1}} f(x) d x \quad \text { and } \quad J^{\prime}\left(\Omega_{1}\right)(\theta)=\int_{\partial \Omega_{1}} f \theta \cdot n d s,
$$

where $n$ is the exterior unit normal to $\partial \Omega_{1}$.

From now on the stacking sequence $\xi$ is fixed and we drop its dependence in all our notations. Consider the linearized buckling problem (4) and (5). For simplicity we restrict ourselves to two phases in a single ply (we refer to Remark 4.5 and [7] for the extension to the case of several plies). Let $\Omega_{1} \Subset \Omega$ be and denote as $n$ as the outer normal to $\Omega_{1}$. We define the complement of $\Omega_{1}$ as $\Omega_{0}=\Omega \backslash \Omega_{1}$. Then the extensional stiffness tensor $\mathcal{A}$ reads

$$
\mathcal{A}=\chi_{1} \mathcal{A}_{1}+\left(1-\chi_{1}\right) \mathcal{A}_{0}=\mathcal{A}_{0}+\chi_{1}\left(\mathcal{A}_{1}-\mathcal{A}_{0}\right)
$$

where $\mathcal{A}_{0}$ and $\mathcal{A}_{1}$ are two different anisotropic elastic laws and $\chi_{1}$ is the characteristic function of $\Omega_{1}$. 
Remark 4.2. When dealing with the multi-layered case, there is a subtle point about the superposition of interfaces in different plies as explained in [7]. Indeed, when one interface is varying in layer $i$, all other ply shapes are fixed and act as a background material in equations (4) and (5). The Hadamard shape sensitivity of partial differential equations works only for smooth coefficients (at least in the vicinity of the interface). Therefore, we have to assume that interfaces in different plies do not superpose. More precisely, we assume that the measure of the common interior interface between two ply shapes $\Omega_{i}, \Omega_{j}$ is negligible, i.e. $\left|\partial \Omega_{i} \cap \partial \Omega_{j}\right|=0, \forall i \neq j=1, \ldots, N$, in order to avoid non-differentiability. Otherwise, moving $\partial \Omega_{i}$ in the direction of its normal or in the opposite direction could yield different directional derivatives, according to the discontinuity of the material properties on both sides in the $j$ layer.

As explained in 17, 23, the formula of the shape derivative for an objective function depending on the solution of (4) or (5) involves jumps of the stress and strain tensors through the interface $\partial \Omega_{1}$. These jump terms are difficult to evaluate numerically, all the more if the interface is not explicitly discretized. Hence, we avoid this delicate issue by calculating the shape derivative of the discretized versions of (4) and (5), which is much easier and is more useful and relevent from a numerical point of view.

We equip $\Omega$ with a conformal simplicial mesh $\Omega_{h}=\bigcup_{i=1}^{M} T_{i}$ with $M$ triangles $T_{i}$ of maximal size $h$. The mesh is fixed and does not fit to the subdomains $\Omega_{0}$ and $\Omega_{1}$. Let $\Pi_{D}^{1}\left(\Omega_{h} ; \mathbb{R}^{2}\right)$ be a finitedimensional approximation of $H_{D}^{1}\left(\Omega ; \mathbb{R}^{2}\right)$, for instance the space of $\mathbb{P}^{1}$ Lagrange finite elements. Define $u_{h} \in \Pi_{D}^{1}\left(\Omega_{h} ; \mathbb{R}^{2}\right)$ as the internal approximations of $u$, solution of

$$
\int_{\Omega_{h}} \mathcal{A} e\left(u_{h}\right): e\left(v_{h}\right) d x=\int_{\Gamma_{N}} g \cdot v_{h} d s, \quad \forall v_{h} \in \Pi_{D}^{1}\left(\Omega_{h} ; \mathbb{R}^{2}\right),
$$

and define the stress tensor $\sigma_{h}=\mathcal{A} e\left(u_{h}\right)$. Note that, as it is defined, $\sigma_{h}$ is not the true stress tensor but just a tensor representing the constitutive equation of the membrane forces per unit length. Nevertheless, by a convenient abuse of notations, we shall call it stress tensor in the sequel. In order to facilitate the analysis, the discretization of the integral functional in 11 is split into two subcases $G_{h, 1}, G_{h, 2}$ given by

$$
G_{h, 1}\left(\Omega_{1}\right)=\int_{\Omega_{h}} s_{1}\left(x, u_{h}\left(\Omega_{1}\right)\right) d x \quad \text { and } \quad G_{h, 2}\left(\Omega_{1}\right)=\int_{\Omega_{h}} s_{2}\left(x, \sigma_{h}\left(\Omega_{1}\right)\right) d x .
$$

The first (smooth) integrand $s_{1}$ depends only on the displacement, while the second one $s_{2}$ depends on the stress $\sigma_{h}=\mathcal{A} e\left(u_{h}\right)$. Both satisfy adequate growth conditions.

Now let $\Pi_{D}^{2}\left(\Omega_{h}\right)$ be a conformal finite-dimensional subspace of $H_{D}^{2}(\Omega)$ (embedded in $C^{1}\left(\Omega_{h}\right)$ ), e.g. Hermite cubic finite element. Let $w_{h} \in \Pi_{D}^{2}\left(\Omega_{h}\right)$ be the internal approximation of $w$, solution of the discretized eigenvalue problem

$$
\int_{\Omega_{h}} E\left(w_{h}, \eta_{h}\right) d x=-\lambda_{h} \int_{\Omega_{h}} B\left(u_{h} ; w_{h}, \eta_{h}\right) d x, \quad \forall \eta_{h} \in \Pi_{D}^{2}\left(\Omega_{h}\right),
$$

where $u_{h}$ solves (28) and $\lambda_{h}$ is the first positive eigenvalue. The bilinear and trilinear forms $E\left(\eta_{1}, \eta_{2}\right)$ and $B\left(v, \eta_{1}, \eta_{2}\right)$ are respectively defined for any $\eta_{1}, \eta_{2} \in \Pi_{D}^{2}\left(\Omega_{h}\right), v \in \Pi_{D}^{1}\left(\Omega_{h} ; \mathbb{R}^{2}\right)$ as

$$
E\left(\eta_{1}, \eta_{2}\right)=\mathcal{D} \nabla^{2} \eta_{1}: \nabla^{2} \eta_{2}, \quad \text { and } \quad B\left(v ; \eta_{1}, \eta_{2}\right)=\mathcal{A} e(v) \cdot \nabla \eta_{1} \cdot \nabla \eta_{2}
$$

The first positive eigenvalue $\lambda_{h}$ of 29 can also be defined through the Rayleigh quotient

$$
\lambda_{h}^{-1}=\max _{\eta \in \Pi_{D}^{2}\left(\Omega_{h}\right)} \frac{-\int_{\Omega_{h}} B\left(u_{h} ; \eta, \eta\right) d x}{\int_{\Omega_{h}} E(\eta, \eta) d x} .
$$

We now state two results on the shape derivatives of these discrite objective functions. Their proofs are briefly sketched and we refer to [7, 23] for more details.

Proposition 4.1. Assume that the interface $\partial \Omega_{1}$ generically cut the mesh $\Omega_{h}$, namely that it is never aligned with part of an edge of any triangle $T_{i}$. Define the discrete adjoint state $p_{h} \in \Pi_{D}^{1}\left(\Omega_{h} ; \mathbb{R}^{2}\right)$ as the solution of

$$
\int_{\Omega_{h}} \mathcal{A} e\left(p_{h}\right): e\left(v_{h}\right) d x=-\int_{\Omega_{h}} \partial_{u} s_{1}\left(x, u_{h}\right) \cdot v_{h} d x, \quad \forall v_{h} \in \Pi_{D}^{1}\left(\Omega_{h} ; \mathbb{R}^{2}\right) .
$$

Then, the solution $u_{h}$ of (28) is shape differentiable and the shape derivative of $G_{h, 1}$ is given by

$$
G_{h, 1}^{\prime}\left(\Omega_{1}\right)(\theta)=\int_{\partial \Omega_{1}} \llbracket \mathcal{A} \rrbracket e\left(u_{h}\right): e\left(p_{h}\right) \theta \cdot n d s,
$$

where $\llbracket \mathcal{A} \rrbracket=\mathcal{A}_{1}-\mathcal{A}_{0}$ denotes the jump of a discontinuous quantity through $\partial \Omega_{1}$. 
Proof. This is a classical result. Given the shape differentiability of $u_{h}$, the shape derivative of $G_{h, 1}$ stems from Céa's fast derivation method [6]. For that purpose, for any $\left(v_{h}, q_{h}\right)$ we introduce the Lagrangian

$$
\mathcal{L}_{h}\left(\theta, v_{h}, q_{h}\right)=\int_{\Omega} s_{1}\left(x, v_{h}\right) d x+\int_{\Omega} \mathcal{A} \circ(I d+\theta)^{-1} e\left(v_{h}\right): e\left(q_{h}\right) d x-\int_{\Gamma_{N}} g \cdot q_{h} d s .
$$

The stationarity conditions

$$
\frac{\partial \mathcal{L}_{h}}{\partial q_{h}}\left(0, u_{h}, p_{h}\right)=0, \quad \frac{\partial \mathcal{L}_{h}}{\partial v_{h}}\left(0, u_{h}, p_{h}\right)=0
$$

yield respectively the state equation for $u_{h}$ and the adjoint equation for $p_{h}$, while the partial shape derivative of $\mathcal{L}_{h}$, evaluated at $u_{h}$ and $p_{h}$, coincides with the shape derivative of $G_{h, 1}$.

Proposition 4.2. Assume the same hypothesis than in Proposition 4.1. Define a new discrete adjoint state $p_{h} \in \Pi_{D}^{1}\left(\Omega_{h} ; \mathbb{R}^{2}\right)$ as the solution of

$$
\int_{\Omega_{h}} \mathcal{A} e\left(p_{h}\right): e\left(v_{h}\right) d x=-\int_{\Omega_{h}} \partial_{\sigma} s_{2}\left(x, \sigma_{h}\right) \cdot \mathcal{A} e\left(v_{h}\right) d x, \quad \forall v_{h} \in \Pi_{D}^{1}\left(\Omega_{h} ; \mathbb{R}^{2}\right) .
$$

The shape derivative of $G_{h, 2}$ is given by

$$
G_{h, 2}^{\prime}\left(\Omega_{1}\right)(\theta)=\int_{\partial \Omega_{1}}\left(\llbracket s_{2}\left(x, \sigma_{h}\right) \rrbracket+\llbracket \mathcal{A} \rrbracket e\left(u_{h}\right): e\left(p_{h}\right)\right) \theta \cdot n d s,
$$

where $\llbracket \cdot \rrbracket=\cdot_{1}-\cdot_{0}$ denotes the jump of a discontinuous quantity through $\partial \Omega_{1}$.

Remark 4.3. The function $s_{2}$ is smooth so the jump $\llbracket s_{2}\left(x, \sigma_{h}\right) \rrbracket$ is due to the jump of the stress tensor $\sigma_{h}$. Recall that the strain tensor $e\left(u_{h}\right)$ is constant in each triangle. Thus the jump of $\sigma_{h}$ is due only to the jump of $\mathcal{A}$.

Proof. The proof is quite similar to that of Proposition 4.1. The only difference lies in the definition of the Lagrangian, which is

$$
\mathcal{L}_{h}\left(\theta, v_{h}, q_{h}\right)=\int_{\Omega} s_{2}\left(x, \mathcal{A} \circ(I d+\theta)^{-1} e\left(v_{h}\right)\right) d x+\int_{\Omega} \mathcal{A} \circ(I d-\theta) e\left(v_{h}\right): e\left(q_{h}\right) d x-\int_{\Gamma_{N}} g \cdot q_{h} d s .
$$

Proposition 4.3. Assume the same hypothesis than in Proposition 4.1. Define another discrete adjoint state $\hat{p}_{h} \in \Pi_{1}\left(\Omega_{h} ; \mathbb{R}^{2}\right)$ as the solution of

$$
\int_{\Omega_{h}} \mathcal{A} e\left(\hat{p}_{h}\right): e\left(v_{h}\right) d x=\lambda_{h} \int_{\Omega_{h}} B\left(v_{h} ; w_{h}, w_{h}\right) d x \quad \forall v_{h} \in \Pi_{D}^{1}\left(\Omega_{h} ; \mathbb{R}^{2}\right) .
$$

Moreover, assume $\lambda_{h}$ is a simple eigenvalue of problem (29) and let the buckling mode $w_{h} \in \Pi_{D}^{2}\left(\Omega_{h}\right)$ be normalized as $\int_{\Omega} B\left(u_{h} ; w_{h}, w_{h}\right) d x=-1$. Then $\lambda_{h}$ is shape differentiable and its shape derivative reads

$$
\lambda_{h}^{\prime}\left(\Omega_{1}\right)(\theta)=\int_{\partial \Omega_{1}}\left(\llbracket \mathcal{D} \rrbracket \nabla^{2} w_{h}: \nabla^{2} w_{h}+\lambda_{h}\left(\llbracket \mathcal{A} \rrbracket e\left(u_{h}\right) \cdot \nabla w_{h}\right) \cdot \nabla w_{h}-\llbracket \mathcal{A} \rrbracket e\left(u_{h}\right): e\left(\hat{p_{h}}\right)\right) \theta \cdot n d s
$$

where $\llbracket \rrbracket=\cdot_{1}-\cdot_{0}$ denotes the jump through $\partial \Omega_{1}$.

Proof. Let us denote $\omega_{i}^{h}(x)$ and $\phi_{i}^{h}(x)$ the basis functions of the finite element spaces $\Pi_{D}^{2}\left(\Omega_{h}\right)$ and $\Pi_{D}^{1}\left(\Omega_{h}, \mathbb{R}^{2}\right)$, respectively. The solutions $w_{h} \in \Pi_{D}^{2}\left(\Omega_{h}\right)$ and $u_{h} \in \Pi_{D}^{1}\left(\Omega_{h}, \mathbb{R}^{2}\right)$ are decomposed as

$$
w_{h}=\sum_{i} W_{i}^{h} \omega_{i}^{h}(x), \quad u_{h}=\sum_{i} U_{i}^{h} \phi_{i}^{h}(x),
$$

and the vectors $W^{h}, U^{h}$ (with respective components $W_{i}^{h}, U_{i}^{h}$ ) are solutions of

$$
E^{h} W^{h}=-\lambda_{h} B^{h} W^{h} \quad \text { and } \quad K^{h} U^{h}=G^{h} .
$$


In (37) the matrices $E^{h}, B^{h}, K^{h}$ and the vector $G^{h}$ are respectively defined as

$$
\begin{array}{rlrl}
E_{i, j}^{h} & =\int_{\Omega_{h}} E\left(\omega_{i}^{h}, \omega_{j}^{h}\right) d x, & B_{i j}^{h} & =\int_{\Omega_{h}} B\left(u_{h} ; \omega_{i}^{h}, \omega_{j}^{h}\right) d x, \\
K_{i, j}^{h} & =\int_{\Omega_{h}}^{\mathcal{A} e\left(\phi_{i}^{h}\right): e\left(\phi_{j}^{h}\right) d x,} \quad G_{i}^{h}=\int_{\Gamma_{N}} g \cdot \phi_{i}^{h} d s .
\end{array}
$$

We remark that the basis functions $\omega_{i}^{h}$ and $\phi_{i}^{h}$ are independent of $\partial \Omega_{1}$. Thus, under the assumption that $\partial \Omega_{1}$ does not overlap any edge of $T_{i} \in \Omega_{h}$, the shape derivatives of $K^{h}, E^{h}$ and $B^{h}$ exist and, according to 30 and 38 , read

$$
\begin{aligned}
& \left(K_{i j}^{h}\right)^{\prime}\left(\Omega_{1}\right)(\theta)=\int_{\partial \Omega_{1}} \llbracket \mathcal{A} \rrbracket e\left(\phi_{i}^{h}\right): e\left(\phi_{j}^{h}\right)(\theta \cdot n) d s, \quad\left(E_{i j}^{h}\right)^{\prime}\left(\Omega_{1}\right)(\theta)=\int_{\partial \Omega_{1}} \llbracket \mathcal{D} \rrbracket \nabla^{2} \omega_{i}^{h}: \nabla^{2} \omega_{j}^{h}(\theta \cdot n) d s \\
& \left(B_{i j}^{h}\right)^{\prime}\left(\Omega_{1}\right)(\theta)=\int_{\partial \Omega_{1}} \llbracket \mathcal{A} \rrbracket e\left(u_{h}\right) \cdot \nabla \omega_{i}^{h} \cdot \nabla \omega_{j}^{h}(\theta \cdot n) d s+\int_{\Omega_{h}} \mathcal{A} e\left(u_{h}^{\prime}\left(\Omega_{1}\right)(\theta)\right) \cdot \nabla \omega_{i}^{h} \cdot \nabla \omega_{j}^{h} d x .
\end{aligned}
$$

It is now a classical result in matrix spectral theory [65] that a simple eigenvalue $\lambda_{h}$ of a differentiable matrix, together with its associated normalized eigenvector $w_{h}$, are differentiable too.

To compute the shape derivative $\lambda_{h}^{\prime}\left(\Omega_{1}\right)(\theta)$, we differentiate the minimak value of the Rayleigh quotient (31) and we use the variational formulation of equation (37) with the derivative of $W^{h}$ as test function. Therefore we obtain

$$
\lambda_{h}^{\prime}\left(\Omega_{1}\right)(\theta)=-\frac{W^{h} \cdot\left(\left(E^{h}\right)^{\prime}\left(\Omega_{1}\right)(\theta)+\lambda_{h}\left(B^{h}\right)^{\prime}\left(\Omega_{1}\right)(\theta)\right) \cdot W^{h}}{W^{h} \cdot B^{h} \cdot W^{h}} .
$$

Choosing $w_{h}$ such that $\int_{\Omega} B\left(u_{h} ; w_{h}, w_{h}\right) d x=W^{h} \cdot B^{h} \cdot W^{h}=-1$, it follows

$$
\begin{aligned}
\lambda_{h}^{\prime}\left(\Omega_{1}\right)(\theta) & =\int_{\partial \Omega_{1}} \llbracket \mathcal{D} \rrbracket \nabla^{2} w_{h}: \nabla^{2} w_{h}(\theta \cdot n) d s+\lambda_{h} \int_{\partial \Omega_{1}} \llbracket \mathcal{A} \rrbracket e\left(u_{h}\right) \cdot \nabla w_{h} \cdot \nabla w_{h}(\theta \cdot n) d s \\
& +\lambda_{h} \int_{\Omega_{h}} \mathcal{A} e\left(u_{h}^{\prime}\left(\Omega_{1}\right)(\theta)\right) \cdot \nabla w_{h} \cdot \nabla w_{h} d x .
\end{aligned}
$$

Finally the desired result arises from the definition of the adjoint state $\hat{p}_{h}$ which allows us to eliminate $u_{h}^{\prime}\left(\Omega_{1}\right)(\theta)$ in the above equation.

Remark 4.4. When $\lambda_{h}$ is not simple, only directional differentiability can be established [21, 65]. In numerical practice we ignore this multiplicity issue and use always the shape derivative of a simple eigenvalue $\lambda_{h}$.

Remark 4.5. The shape derivatives for a single ply were developed in this section. The multi-layered case or equivalently the partial derivatives with respect to each layer $i$, follow directly from the same formulae by adding the index $i=1, \ldots, N$ in the jump formulae (33), (35) and (36). More precisely, we replace $\llbracket \mathcal{A} \rrbracket$ by $\left(\mathcal{A}_{i}-\mathcal{A}_{0}\right)$ (in numerical practice we further take the weak material $\left.\mathcal{A}_{0}=0\right)$. We do the same for the jumps of the bending stiffness tensor $\mathcal{D}$. When the boundaries of some ply shapes coincide, we completely ignore the non-differentiability issue evoked in Remark 4.2. In numerical practice we always use the same derivative formulae (33), (35) and (36), which can be interpreted as "smoothing" the discontinuity of the elastic properties.

\subsection{Shape representation by the level set method}

Consider $\Omega \subset \mathbb{R}^{2}$ a bounded domain in which all admissible shapes are included. In numerical practice, the domain $\Omega$ will be uniformly meshed once and for all. We apply the level set method of Osher and Sethian 63 to implicitly represent the collection of ply shapes $\Omega_{i} \subset \Omega$ of the composite laminate. We define the set of level set functions $\left\{\psi_{i}\right\}_{1 \leq i \leq N}$ such that

$$
\left\{\begin{array}{l}
\psi_{i}(x)=0 \quad \Longleftrightarrow x \in \partial \Omega_{i} \\
\psi_{i}(x)>0 \quad \Longleftrightarrow x \in \Omega_{i} \\
\psi_{i}(x)<0 \quad \Longleftrightarrow x \in \Omega \backslash \bar{\Omega}_{i}
\end{array}\right.
$$

The normal $n_{i}$ of each phase is recovered as $\nabla \psi_{i} /\left|\nabla \psi_{i}\right|$, which also define a natural extension of $n_{i}$ to the whole domain $\Omega$. The level set function $\psi_{i}$ is related to the characteristic function of the shape $\Omega_{i}$ 
by the relation $\chi_{i}=\mathbb{H}\left(\psi_{i}\right)$, where $\mathbb{H}$ is an approximation of the Heaviside function [67]. Therefore, the knowledge of the level set functions completely characterizes the material tensors (1) and (2) or (3).

During the optimization process, the set of shapes $\left\{\Omega_{i}(t)\right\}_{i}$ are going to evolve according to a fictitious time $t \in \mathbb{R}^{+}$, which corresponds to the descent stepping. As it is well known, the evolution of the level set function $\psi_{i}$ is governed by a Hamilton-Jacobi equation

$$
\frac{\partial \psi_{i}}{\partial t}+\mathcal{V}_{i}\left|\nabla \psi_{i}\right|=0
$$

where $\mathcal{V}_{i}$ is the normal velocity. Equation $(39)$ is posed in the whole domain $\Omega$, and not only on the interface $\partial \Omega_{i}$. The main point in using a level set method is that it replaces the Lagrangian evolution of the boundary $\partial \Omega_{i}$ by the Eulerian solution of a transport equation in the whole fixed domain $\Omega$, easily allowing topology changes. In practice the Hamilton-Jacobi equation $\sqrt{39}$ is solved by an explicit second order upwind scheme (see e.g. [67]) on a fixed Cartesian grid. The boundary conditions for $\psi_{i}$ are of Neumann type. Since this scheme is explicit in time, its time stepping must satisfy a CFL condition. Moreover, in order to regularize the level set function (which may become too flat or too steep), we reinitialize it periodically by solving another Hamilton-Jacobi equation which admits as a stationary solution the signed distance to the initial interface [67].

The choice of the normal velocities $\left\{\mathcal{V}_{i}\right\}_{i}$ in $[39]$ is based on the shape derivatives computed in the previous subsection [8]. Generally speaking, the shape derivative of a functional $J\left(\Omega_{i}\right)$ (keeping the other ply shapes fixed) in the direction $\theta_{i} \in W^{1, \infty}\left(\Omega ; \mathbb{R}^{2}\right)$ reads

$$
J^{\prime}\left(\Omega_{i}\right)\left(\theta_{i}\right)=\int_{\partial \Omega_{i}} \mathcal{T}_{i} \theta_{i} \cdot n_{i} d s
$$

where the integrand $\mathcal{T}_{i}(x)$ depends on the solutions of the plate model (4)-(5) and possibly of some adjoint equation. Note that each ply shape $\Omega_{i}$ can move with its own velocity. Since only the normal component of $\theta_{i}$ plays a role in 40, , a descent direction for $J$ is just a collection of vector fields $\Theta=\left\{\theta_{i}\right\}_{i}$ satisfying

$$
\theta_{i}=\mathcal{V}_{i} n_{i} \quad \text { and } \quad J^{\prime}\left(\Omega_{i}\right)\left(\theta_{i}\right)=\int_{\partial \Omega_{i}} \mathcal{T}_{i} \mathcal{V}_{i} d s \leq 0
$$

To ensure the decrease of $J$, the simplest choice is $\mathcal{V}_{i}=-\mathcal{T}_{i}$. However, $\mathcal{T}_{i}$ is a priori defined only on the interfaces $\partial \Omega_{i}$ while $\mathcal{V}_{i}$ must be defined in the entire domain $\Omega$. Therefore, the choice $\mathcal{V}_{i}=-\mathcal{T}_{i}$ is implicitly depending on some extension process of $\mathcal{T}_{i}$. If such an extension is not obvious (as is the case in the presence of jumps in formulae like (33), (35) and (36)), or if we want to regularize the velocity fields, there is an alternate choice based on a different underlying scalar product (see e.g. [8], [21]). For a given lengthscale $\delta>0$ (typically $\delta$ is chosen to be proportional to the mesh size $h$ ), we introduce the following scalar product on $H^{1}(\Omega)$

$$
\left\langle\mathcal{V}_{i}, V\right\rangle_{H^{1}}=\int_{\Omega}\left(\mathcal{V}_{i} V+\delta^{2} \nabla \mathcal{V}_{i} \cdot \nabla V\right) d x
$$

Then, we can compute the normal descent direction $\mathcal{V}_{i} \in H^{1}(\Omega)$ as the unique solution of

$$
\left\langle\mathcal{V}_{i}, V\right\rangle_{H^{1}}=-J^{\prime}\left(\Omega_{i}\right)\left(V n_{i}\right), \quad \forall V \in H^{1}(\Omega) .
$$

One can easily check that the optimal choice $V=\mathcal{V}_{i}$ guarantees a descent direction $J^{\prime}\left(\Omega_{i}\right)\left(\mathcal{V}_{i} n\right) \leq 0$. The variational formulation 42 is both a regularization and an extension of the original shape derivative $J^{\prime}\left(\Omega_{i}\right)$.

In numerical practice an additional ingredient is the approximation of the surface integral 40 as a volume integral

$$
\int_{\partial \Omega_{i}} \mathcal{T}_{i} V d s \approx \int_{\Omega} \delta_{\partial \Omega_{i}} \mathcal{T}_{i} V d x
$$

where the Dirac mass $\delta_{\partial \Omega_{i}}$ is approximated by

$$
\delta_{\partial \Omega_{i}} \approx \frac{1}{2}\left|\nabla s_{\varepsilon}\left(\psi_{i}\right)\right|
$$

where $s_{\varepsilon}$ is an approximation of the sign function

$$
s_{\varepsilon}\left(\psi_{i}\right)=\frac{\psi_{i}}{\sqrt{\psi_{i}^{2}+\varepsilon^{2}}},
$$

where $\varepsilon>0$ is a small parameter chosen in order to spread the integration over a few cells around the interface $(\varepsilon \approx h)$. 


\section{Numerical results}

\section{$5.1 \quad$ Test case description}

The goal of our test cases is to design the lightest composite fuselage skin panel, subject to a shear load, as illustrated in Figure 5. Of particular interest is the study of the influence of the orthotropic plies oriented at $45^{\circ}$ and $-45^{\circ}$ in the prevention of buckling. The objective function is either 10 ) (the weight of the structure) or (13) (a combination of weight and perimeter with $\gamma=0.1 \max (\Delta x, \Delta y)$ ), constrained by different stiffness measures of the type of [11), including compliance, the exact buckling load $\lambda_{1}^{-1}$ or the approximate buckling load (reserve factor) $\lambda_{R F}^{-1}$. We sometimes chose $(13)$, which features a perimeter term, instead of (10), as objective function in order to enforce some smoothness of the optimal shapes. Furthermore a compliance constraint is always included since it enhances the overall stiffness of the structure.

Due to the small curvature of the cylindrical panel section, an approximative plate model is used. In the sequel we use adimensionalized units for all physical quantities. The panel domain is a rectangle $\Omega=[0,2] \times[0,1]$, modeled as a multi-layered plate, in accordance with Configuration I detailed in Section 2 (see Figure 2). In other words, each ply is composed of two phases, one stiff $\mathcal{A}_{i}$ and one weak $\mathcal{A}_{0}$, mimicking "holes". For numerical purposes the tensor $\mathcal{A}_{0}$ is equal to the main material of each ply multiplied by a factor $10^{-3}$.

The panel boundary conditions are described in Figure 5 , namely $\Omega$ is clamped on its left side $\Gamma_{D}$, the upper and lower boundaries are free and a vertical in-plane load $g$ is applied on the right side. There are actually two slightly different load cases. The first load case is a surface load $g=(0,-0.5)$, applied on the segment $x=2, y=[0.4,0.6]$. The second load case is a surface load $g=(0,-0.05)$, applied on the entire right boundary of $\Omega$.

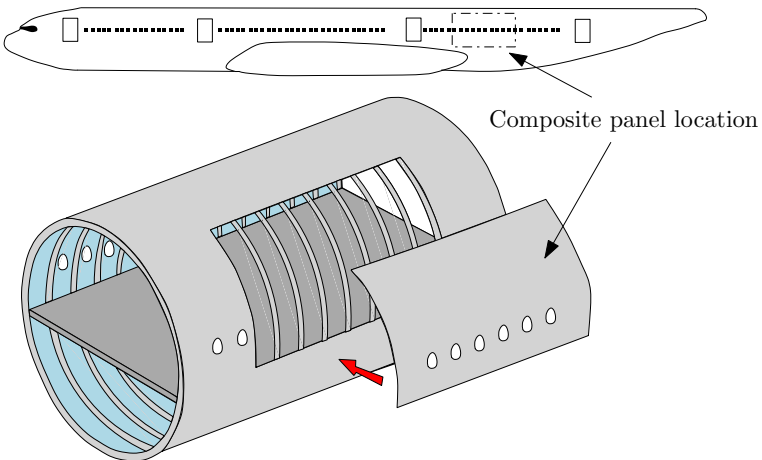

(a) Fuselage panel

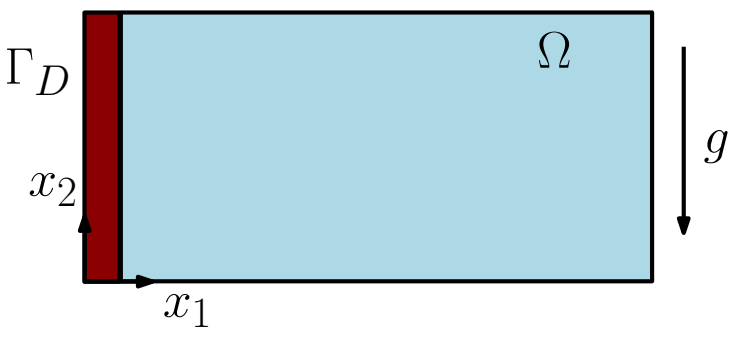

(b) Approximative flat model due to the small curvature of the cylindrical panel section

Figure 5: Composite test case.

The elastic properties of the main phase of each layer are described through one of the following tensors: $\mathcal{C}_{0^{\circ}}, \mathcal{C}_{90^{\circ}}, \mathcal{C}_{45^{\circ}}, \mathcal{C}_{-45^{\circ}}$. Each of them corresponds to the rotation of an orthotropic material $\mathcal{C}$, whose $2 \mathrm{D}$ components can be expressed via the Voigt matrix notation

$$
\mathcal{C}=\frac{1}{1-\nu_{x y} \nu_{y x}}\left(\begin{array}{ccc}
E_{x} & \nu_{y x} E_{x} & 0 \\
\nu_{x y} E_{y} & E_{y} & 0 \\
0 & 0 & 2 G_{x y}
\end{array}\right)
$$

where $E_{x}, E_{y}$ are the Young modulus in the orthotropy directions, $\nu_{x y}$ is the Poisson's ratio, $G_{x y}$ is the shear modulus and $\nu_{y x} E_{x}=\nu_{x y} E_{y}$. Each tensor $\mathcal{C}_{\alpha}, \alpha=0^{\circ}, 90^{\circ}, 45^{\circ},-45^{\circ}$, can be characterized through a rotation of the tensor $\mathcal{C}$ according to an angle $\alpha$ by $\mathcal{C}_{\alpha}=\mathcal{Q}^{T}(\alpha) \mathcal{C} \mathcal{Q}(\alpha)$ with

$$
\mathcal{Q}(\alpha)=\left(\begin{array}{ccc}
\cos ^{2}(\alpha) & \sin ^{2}(\alpha) & \sqrt{2} \sin (\alpha) \cos (\alpha) \\
\sin ^{2}(\alpha) & \cos ^{2}(\alpha) & -\sqrt{2} \sin (\alpha) \cos (\alpha) \\
-\sqrt{2} \sin (\alpha) \cos (\alpha) & \sqrt{2} \sin (\alpha) \cos (\alpha) & \cos ^{2}(\alpha)-\sin ^{2}(\alpha)
\end{array}\right) .
$$

The elastic parameters of the tensor $\mathcal{C}$ are normalized to Young moduli $E_{x}=1$., $E_{y}=0.05$, shear modulus $G_{x y}=0.03$ and Poisson ratio $\nu_{x y}=0.3$. These values approximatively match the elastic parameter proportions of carbon fiber/epoxy. The thickness of each ply is fixed to $\varepsilon=0.125$. 
Our numerical test case is quite simplified to focus on the main difficulties (mixing topology optimization of the ply shapes and discrete stacking sequence optimization) although it is truly representative of real aeronautic cases. One important simplification is that we assume a flat composite panel in order to use a plate model. It would be not very hard to consider a cylindrical panel and a shell model which is easily map to a plane computational domain. We also consider simplified boundary conditions but there is no conceptual difficulties in treating more general ones.

The in-plane elastic and adjoint problems are solved via $\mathbb{P}^{1}$ Lagrange finite elements, meanwhile the bending problem is solved with Morley finite elements (non-conforming piecewise $\mathbb{P}^{2}$ ). In Section 4 the shape derivative was computed for conforming finite elements. In numerical practice we use the same formulas, even for the non-conforming Morley finite elements. The finite element computations are performed with the Freefem ++ software [28, 34].

\subsection{Optimization algorithm}

The optimization algorithm for solving (9) can be summarized as two nested loops.

1. An outer loop for the shape variable $\mathcal{O}$ that solves $(9)$ for a fixed stacking sequence $\xi$ via a descent direction method, which is based on a shape sensitivity analysis coupled to a level set method described in Subsection 4.2

2. An inner loop for the variable $\xi$ where the constraint margin function $\mathcal{M}(\mathcal{O})$ is evaluated by solving the integer programming problem (17) via Algorithm 3.1.

Let $N_{\text {stack }}$ be the frequency at which the lower level (or inner loop) is solved and $\epsilon>0$ the stopping criterion tolerance. We propose the following iterative optimization algorithm for the composite design problem

Algorithm 5.1. Initialize $\mathcal{O}^{0} \in \mathcal{U}_{\text {ad }}$ through a collection of level set functions $\left\{\psi_{i}^{0}\right\}_{i}$ representing each layer, defined as the signed distance function of a chosen initial topology. Evaluate the constraint margin function $\mathcal{M}\left(\mathcal{O}^{0}\right)$ in $(17)$ and define the initial stacking sequence $\xi^{0}$ as its optimal solution.

$\left\{\begin{array}{l}\text { For } k \geq 0 \\ \end{array}\right.$

1) Compute $u^{k}, \lambda_{1}^{k}$ and $w_{1}^{k}$ by solving problems (4) and (5) for a fixed $\xi^{k}$. If the stiffness criterion is compliance or $\lambda_{R F}$, then only compute $u^{k}$.

2) Evaluate in accordance to Section 4 the discrete shape derivatives $J_{h}^{\prime}$ and $G_{h}^{\prime}$ in (9) for each layer $1 \leq i \leq N$.

3) Define a set of descent velocities $\Theta^{k}=\left\{\mathcal{V}_{i}^{k} n_{i}^{k}\right\}_{i}$ and advect the level set functions $\psi_{i}^{k}$, as described in Subsection 4.2. The pseudo-time step $\Delta t^{k}$ is chosen so that, for $\mathcal{O}^{k+1}=\left(I d+\Delta t^{k} \Theta^{k}\right) \mathcal{O}^{k}, \quad J\left(\mathcal{O}^{k+1}, \xi^{k}\right) \leq J\left(\mathcal{O}^{k}, \xi^{k}\right)$ and $\mathcal{M}\left(\mathcal{O}^{k+1}, \xi^{k}\right) \leq 0$.

4) If $k$ is a multiple of $N_{\text {stack }}$, compute $\xi^{k+1}$ which minimizes $\mathcal{M}\left(\mathcal{O}^{k+1}\right)$ via the outer approximation (OA) method. Otherwise set $\xi^{k+1}=\xi^{k}$.

5) If $\left|J\left(\mathcal{O}^{k+1}\right)-J\left(\mathcal{O}^{k}\right)\right| \leq \epsilon$, then STOP. Otherwise return to 1$)$.

Before going to the numerical results, we discuss some issues on the numerical practice of the above algorithm.

Remark 5.1. (Descent direction in step 3).) It is obtained by a method of feasible direction (see e.g. [77] ; we borrow an implementation from [5]) for the following constrained problem (with given stacking sequence $\xi^{k}$ )

$$
\left\{\begin{array}{l}
\min _{\mathcal{O} \in \mathcal{U}_{a d}} J(\mathcal{O}) \\
\text { such that } G\left(\mathcal{O}, \xi^{k}\right) \leq 0 .
\end{array}\right.
$$

In short, the method of feasible direction works like a sequential linear programming approximation method. 
Remark 5.2. (Combinatorial optimization in step 4).) The optimal $\xi^{k+1}$ is found by means of the MILP routine of the commercial software CPLEX ${ }^{\circledR}$ (IBM-ILOG) [40] for MATLAB ${ }^{\circledR}$ [54]. The frequency is taken as $N_{\text {stack }}=5$. Actually, we limit the total number of combinatorial optimizations of the constraint margin function to 10. After that, the stacking sequence is kept fixed.

Remark 5.3. (Stopping criterion in step 5).) Strictly speaking, such a naive stopping criterion does not ensure convergence to an optimal solution. One should rather impose a criterion based on the norm of the gradient. However, it is sufficient for our test cases in numerical practice.

Remark 5.4. (Initialization of the algorithm.) For all numerical applications below, all plies are initialized with the same shape. The initial stacking sequence $\xi^{0}$ is computed in two steps. First, we optimize the constraint margin function which takes into account only the compliance (and thus ignoring other constraints such as buckling): it yields a stacking sequence $\xi^{*}$. As a matter of fact, this is a convex integer programming problem, according to Proposition 3.2, and the OA algorithm can exactly solve it. Second, we fix the fiber orientation proportions given by $\xi^{*}$ and we evaluate a new constraint margin function taking into account only the bending constraint (and not the compliance). This is again a convex problem which can exactly be solved by the OA algorithm, by virtue of Proposition 3.3. The resulting stacking sequence is our initial $\xi^{0}$.

Remark 5.5. (Stacking sequence optimization for multiple constraints.) When dealing with several constraints, according to equation (17), the constraint margin function involves the minimization of the maximal constraint. Solving such a min-max problem may turn to be numerically costly. Therefore, for the sake of efficiency, we approximate the constraint margin function via the following heuristic. First, we evaluate the constraint margin functions associated to each constraint. Second, we solve the min-max problem for the restricted set of stacking sequence which were optimal in the first step. If the constraint margin function evaluated at this solution is negative, we are fine. However if it is positive, it is of no use and thus we keep as optimal stacking sequence the previous one obtained at the previous iteration. This is of course just one possible heuristic to approximatively evaluate the constraint margin function and not necessarily the most efficient.

\subsection{Mass minimization with a compliance constraint}

Our first test case is a compliance problem with no buckling constraint:

$$
\left\{\begin{array}{l}
\min _{\mathcal{O} \in \mathcal{U}_{a d}} J(\mathcal{O})=\sum_{i=1}^{4} \int_{\Omega} \chi_{i} d x \\
\text { such that } \int_{\Omega} \mathcal{A} e(u): e(u) d x \leq C_{0},
\end{array}\right.
$$

where $\mathcal{O}$ is a 4 -layered composite with fiber orientations $0^{\circ}, 90^{\circ}, 45^{\circ},-45^{\circ}$. The only state equation is the elasticity system (5). We remark that since each fiber orientation must be present in the laminate and their order within the stacking is irrelevant for the extensional stiffness tensor $\mathcal{A}$, the stacking sequence variable $\xi$ does not play any role here. However, the tensor $\mathcal{A}$, as well as the solution $u$ of (5), depend on $\mathcal{O}$. The constant $C_{0}$ is equal to twice the initial compliance of the structure and the load $g=(0,-0.5)$ is defined according to Figure 5 (b) throughout the right boundary of $\Omega$. The computational domain $\Omega$ is meshed with $30 \times 60$ square elements (each of them divided in two triangles). The initialization and the optimal results of the level-set method are displayed on Figure 6. In all our figures, the black color stands for the material meanwhile white is "void".

For the sake of comparison we show in Figure 7 the optimal results obtained with the SIMP method (more precisely with the software OptiStruct ${ }^{\circledR}$ ). The red and blue colors represent the highest and lowest densities of material, respectively (note that there are still yellow or green intermediate densities which did not disappear although they are penalized). The results for the $90^{\circ}$ and $0^{\circ}$ orientations are very similar, while they are coarser and smoother for the $-45^{\circ}$ and $45^{\circ}$ orientations in the level set case, nevertheless with roughly the same geometrical orientation of the subdomains. We do not compare the precise designs obtained by each method, which are quite different, and the performances of which are even more difficult to compare since intermediate densities are still present in the SIMP result. Of course, it is highly possible that some other SIMP software, with a better penalization process, produces optimal designs with less intermediate densities. 

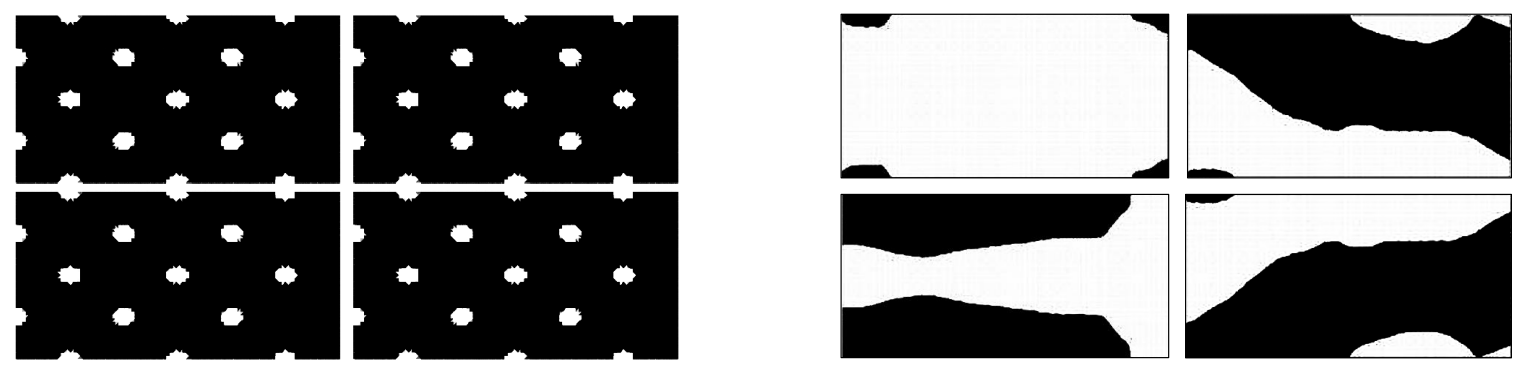

Figure 6: Numerical results of Subsection 5.3. initial configuration (left) and optimal design (right). The fiber orientations are: $90^{\circ}$ upper-left, $0^{\circ}$ lower-left, $-45^{\circ}$ upper-right and $45^{\circ}$ lower-right.
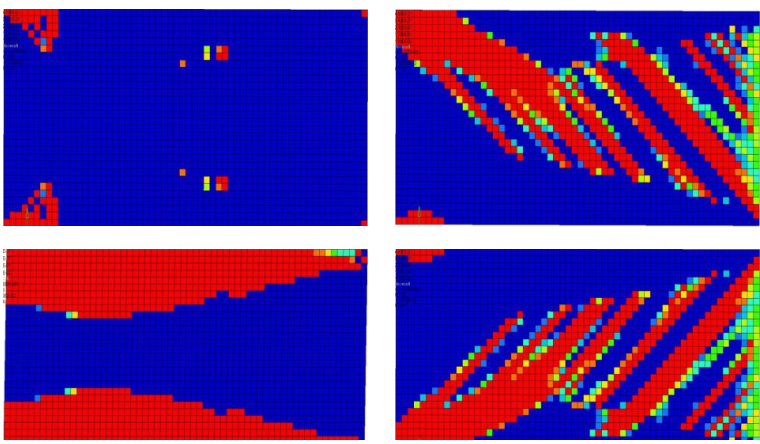

Figure 7: Results obtained with the SIMP method for the test case of Subsection 5.3 . The fiber orientations are: $90^{\circ}$ upper-left, $0^{\circ}$ lower-left, $-45^{\circ}$ upper-right and $45^{\circ}$ lower-right.

\subsection{Mass minimization with a bending constraint: $\lambda_{1}$}

Our second test case is the more involved one since it relies on the two state equations (4) and (5):

$$
\left\{\begin{array}{l}
\min _{\mathcal{O} \in \mathcal{U}_{a d}, \xi \in \mathcal{Y}_{a d}} J(\mathcal{O})=\sum_{i=1}^{8}\left(\rho \int_{\Omega} \chi_{i} d x+\gamma \operatorname{Per}\left(\Omega_{i}\right)\right) \\
\text { such that } \int_{\Omega} \mathcal{A} e(u): e(u) d x \leq C_{0}, \\
\text { and } \lambda_{1}^{-1}(\mathcal{O}, \xi) \leq 1,
\end{array}\right.
$$

where $(\mathcal{O}, \xi)$ is a 8-layered composite laminate with stacking sequence $\xi$ and in-plane ply shapes $\mathcal{O}$. Recall that the layered composite is symmetrized so that the total number of plies is actually 16 . Of course, the tensor $\mathcal{A}$, as well as the solution $u$ of $(5)$, depend on $(\mathcal{O}, \xi)$. The minimal proportion of plies in each orientation is 1 out of 8 (constraint (R4) in Section 3). The constant $C_{0}$ is equal to ten times the initial compliance of the structure. The load $g=(0,-0.5)$ is defined according to Figure 5 (b) and is applied on the segment $x=2, y=[0.4,0.6]$. The computational domain $\Omega$ is meshed with $50 \times 100$ square elements (each of them divided in two triangles). The optimal results are shown on Figure 8 . We plot, not only the shape of each ply, but also the sum of all ply characteristic functions, corresponding to the total density of the composite plate. Indeed, none of the ply shapes does connect the load to the supporting wall, but of course their union does so. We remark also the presence of the four fiber orientations within the laminate thanks to the imposed constraint on their minimal proportions. The convergence history is shown on Figure 9 the two inequality constraints are active at convergence.

Remark 5.6. In sub-figure (d) of Figure 8 the regions of high density look thicker than the superposition of plies in sub-figure (e): this is due to the smoothing effect when passing from the level set functions to the densities $\left(\chi_{i}=\mathbb{H}\left(\psi_{i}\right)\right.$, where $\mathbb{H}$ is an approximate Heaviside function, see Section 4.2). The same will be true for all subsequent figures in this section.

Remark 5.7. During the optimization process of this second test case, the most inner layers have a tendency to become almost void while all the mass is concentrated on the outer layers. This makes sense in view of an analogy with an Euler-Bernoulli beam: the outer layers contribute more to the inertia moment 
of the structure than the inner ones. Hence they are preferable to avoid buckling. Additionally, due to the vertical direction of the surface load $g$, the principal stress lines promote the use of reinforced fibers in the directions $0^{\circ}$ and $45^{\circ}$, which tends to eliminate the rest of reinforcement orientations (especially $\left.90^{\circ}\right)$. Note that the constraint (R4) on the minimal ply proportion in each orientation does not prevent some plies to be "filled" by the weak material $\mathcal{A}_{0}$ and thus some orientations of the orthotropic material are not present in the end.

This absence might be undesirable when e.g. we expect the total mass of the structure to be distributed "uniformly" through all fiber orientations in the laminate composite. In order to limit the above bias, once the stacking sequence is fixed (namely after the maximal 10 updates of the stacking sequence), the density $\rho$ of each fiber orientation is changed from its initial value $\rho=1$ in order to favor the fiber orientation $-45^{\circ}$ and $90^{\circ}$ in the objective function. More precisely, after 40 iterations we take $\rho=0.05$ for $-45^{\circ}, \rho=0.1$ for $90^{\circ}$, while keeping $\rho=1$ for $45^{\circ}$ and $0^{\circ}$. We will apply the same procedure in the next subsection (except otherwise mentioned).

In the optimization process, we always assume that the first eigenvalue $\lambda_{1}$ is simple, so that we can use the shape derivative result of Proposition 4.3. Otherwise, in the case of eigenvalues crossing, a different shape derivative and optimization algorithm should be used. We checked that $\lambda_{1}$ is indeed simple in our numerical tests. As can be seen on Figure 9, during the evolution of the algorithm the values of $\lambda_{1}^{-1}$ and $\lambda_{2}^{-1}$ never coincide (even though they seem quite close at the 18 th iteration with $\lambda_{1}^{-1}=0.477$ and $\left.\lambda_{2}^{-1}=0.455\right)$. At the optimal shape $\lambda_{1}^{-1}=1$ and $\lambda_{2}^{-1}=0.88$.

\subsection{Mass minimization with a bending constraint: $\lambda_{R F}$}

Our third and final test case is a simplification of the previous one where the true buckling load is replaced by the so-called reserve factor. The mesh is the same as in the previous subsection. This problem is much simpler than the previous one since no buckling analysis (4) is required. In other words, the elasticity system (5) is the only state equation. For the same number of plies, it reads:

$$
\left\{\begin{array}{l}
\min _{\mathcal{O} \in \mathcal{U}_{a d}, \xi \in \mathcal{Y}_{a d}} J(\mathcal{O})=\sum_{i=1}^{8}\left(\rho \int_{\Omega} \chi_{i} d x+\gamma \operatorname{Per}\left(\Omega_{i}\right)\right) \\
\text { such that } \int_{\Omega} \mathcal{A} e(u): e(u) d x \leq C_{0}, \\
\text { and } \mathcal{X}_{R F}(\mathcal{O}, \xi) \leq 1,
\end{array}\right.
$$

where $\mathcal{X}_{R F}$ is a $L^{p}$-approximation of $\lambda_{R F}^{-1}$, defined by

$$
\mathcal{X}_{R F}(\mathcal{O}, \xi)=\left(\int_{\Omega} \frac{\left|(\mathcal{A} e(u))_{x x}^{-}\right|^{p}}{\left(\frac{2 \pi^{2}}{b^{2}}\left(\sqrt{\mathcal{D}_{11} \mathcal{D}_{22}}+\mathcal{D}_{12}+2 \mathcal{D}_{33}\right)\right)^{p}} d x\right)^{1 / p} .
$$

Indeed, as $p$ tends to $+\infty, \mathcal{X}_{R F}$ converges to $\lambda_{R F}^{-1}$ which, according to $(14)$, is given by

$$
\lambda_{R F}^{-1}(\mathcal{O}, \xi)=\left\|\frac{\left|(\mathcal{A} e(u))_{x x}^{-}\right|}{\left(\frac{2 \pi^{2}}{b^{2}}\left(\sqrt{\mathcal{D}_{11} \mathcal{D}_{22}}+\mathcal{D}_{12}+2 \mathcal{D}_{33}\right)\right)}\right\|_{L^{\infty}(\Omega)} .
$$

The advantage of this $L^{p}$-regularization is that $\mathcal{X}_{R F}$ is a differentiable function while $\lambda_{R F}^{-1}$ is not (cf. Remark 2.7. . We take $p=2$ and $p=6$, ensuring in this way the differentiability of $\mid \mathcal{A} e(u))_{x x}^{-} \mid$at 0 (in fact we could have taken any exponent $p \geq 2$ ). The need of adding a compliance constraint to the problem, is due to the very definition of $\lambda_{R F}$, which penalizes only the compressed zones inside the structure, allowing the generation of zones under critical positive tensile strains (which indicate that the structure is close to break).

We consider the first load case, as described in subsection 5.1. with a constraint level $C_{0}$ equal to ten times the initial compliance. The numerical results and the convergence curves are displayed in Figure 10 for $p=2$ and Figure 11 for $p=6$, respectively. The final results in Figure 10 (d) and Figure 11 (d) look very similar and share the same stacking sequence, although the details of each individual layer are different. The convergence history is displayed on Figure 12, As expected the number of iterations until convergence is higher for larger values of the exponent $p$. 


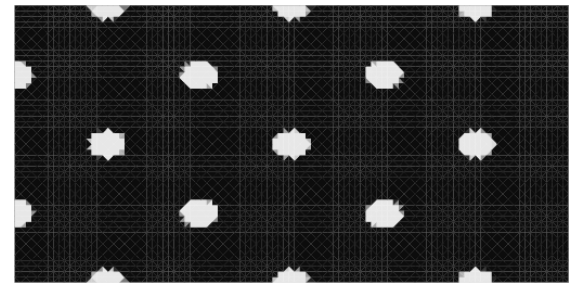

(a) Total density, iteration 0

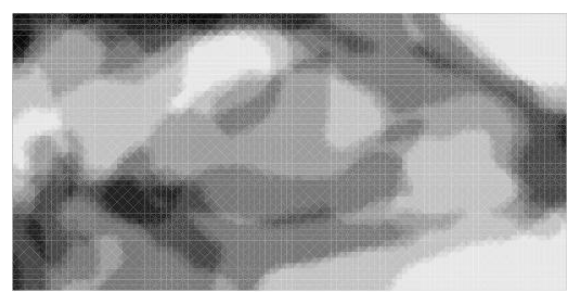

(c) Total density, iteration 10

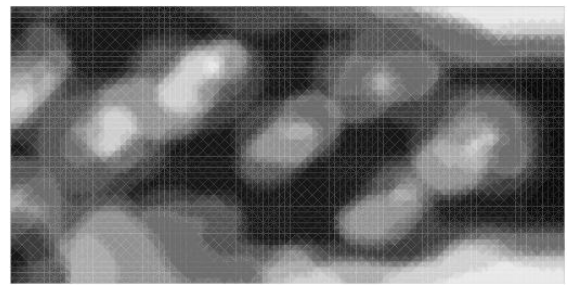

(b) Total density, iteration 5

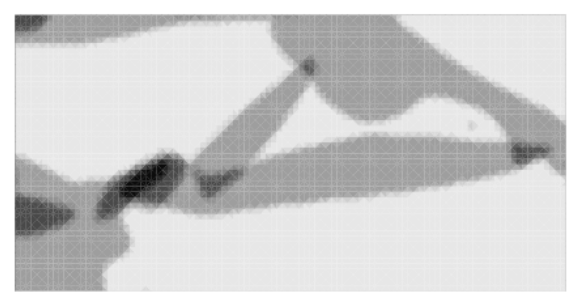

(d) Final total density (iteration 80)

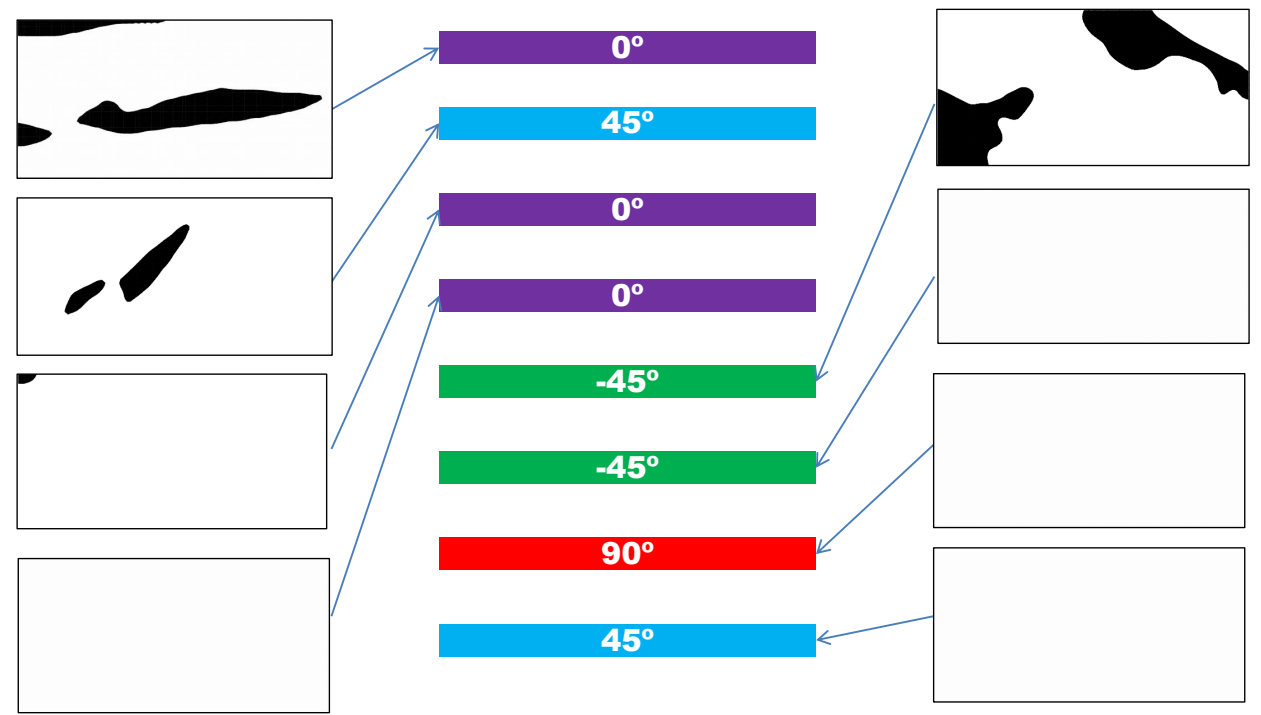

(e) Optimal stacking sequence and shape of each ply. The outer layers are upper in the figure.

Figure 8: Numerical results of Subsection 5.4 (a),(b),(c),(d): Evolution of the total density. (e): Optimal stacking sequence and in-plane shape of each ply. 


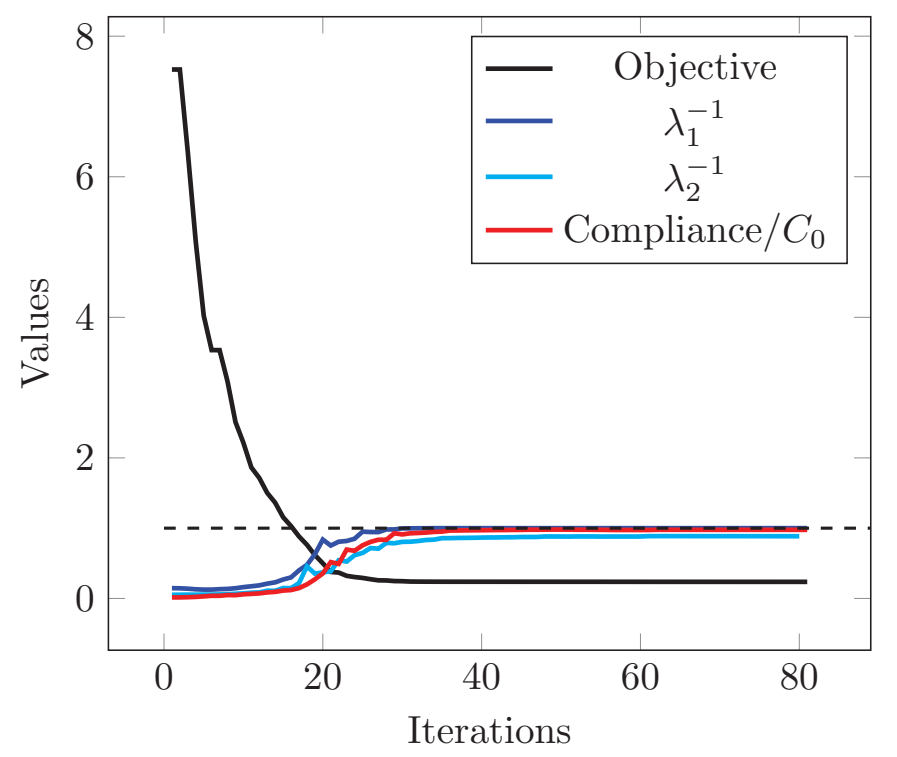

Figure 9: Convergence history, in terms of the iteration number, for the test case of Subsection 5.4 .

For the optimal shape with $p=2$, we computed $\lambda_{1}=0.17$, meanwhile for $p=6, \lambda_{1}=0.28$. Theoretically the laminate should thus buckle since $\lambda_{1}<1$. This phenomenon is explained since, first, $\lambda_{R F}$ is not a good approximation of $\lambda_{1}$ and, second, the exponent $p$ is not large enough to ensure that $\mathcal{X}_{R F}$ is a good numerical approximation of $\lambda_{R F}^{-1}$. As a matter of fact, $\mathcal{X}_{R F}$ is close to $\lambda_{R F}^{-1} \approx 2.3$ when $p \approx 1000$.

We then study the second load case of subsection 5.1. with $p=6$ and a constraint level $C_{0}$ defined as five times the initial compliance. In Figure 13 we perform the computation with our usual ponderation rule for the layer densities, as explained in Remark 5.7. The result is somehow deceiving since the inner layers are full. Therefore, we re-do this optimization with an equal material density in each layer and the result in Figure 14 turns out to be quite different. This sensitivity of the optimal design to the choice of material densities shows that the reserve factor $\lambda_{R F}$ (which is merely a crude approximation of the bubkling load) is not a very robust mechanical criterion in shape optimization. In any case, the optimal design favors the fiber orientations $45^{\circ}$ and $-45^{\circ}$. The convergence history is displayed in Figure 15 . For all the computations of the present subsection, the two inequality constraints are active at convergence.

For the first optimal shape we computed $\lambda_{1}=0.2$, while for the second one $\lambda_{1}=1.71$. These values are different from $\lambda_{R F}^{-1} \approx 2.2$ for both cases. The reason is the same as explained for the first load case.

\subsection{Comparison of the true and approximate buckling factors}

The reserve factor $\lambda_{R F}$ is an approximation of the true buckling factor $\lambda_{1}$ which is popular in engineering practice. It yields a drastic reduction of CPU time for the optimization process since the state equation is just linearized elasticity. However, it is not clear that it is a good approximation since it is based on the assumption of a large aspect ratio, homogeneous plate, which is not satisifed in our shape optimization setting. To check the adequation of $\lambda_{R F}$ as an approximation of $\lambda_{1}$, we make a comparison of both quantities for the test case of Subsection 5.4. Recall that $1 / \lambda_{1}$ is plotted on Figure 9 and remains bounded from above by 1 . As in the previous subsection we approximate $1 / \lambda_{R F}$ by $\mathcal{X}_{R F}$ with $p=2$. To avoid singularities which occur when a hole appears in the optimal design, implying that $\mathcal{D}$ vanishes, we integrate only on the non empty part of the plate $\Omega$ in the definition of $\mathcal{X}_{R F}$. We plot $\mathcal{X}_{R F}$, on the one hand, and the ratio $\lambda_{1}^{-1} / \mathcal{X}_{R F}$, on the other hand, in Figure 16 . Note that there is a bump in the $\mathcal{X}_{R F}$ and $1 / \lambda_{1}$ curves around iteration 20 , which corresponds to a change of topology.

Let us remark that the values of the reserve factor $\lambda_{R F}$, defined by (14), are very different from those of $\lambda_{1}$ (by a factor of at least 20) which is not a surprise since the laminated plate is far from being homogeneous. Even worse is the fact that the ratio is neither constant, nor monotone. At convergence its value is close to $1 / 20$. Therefore, we believe the reserve factor $\lambda_{R F}$ is not an adequate approximation of the true buckling factor $\lambda_{1}$ for shape optimization of non-homogeneous multi-layered composites.

Acknowledgments. The authors would like to thank Airbus Group for its financial support in the 


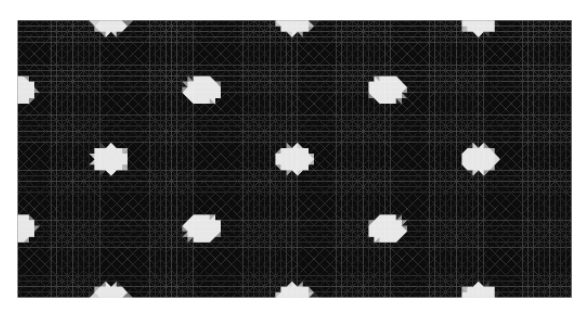

(a) Total density, iteration 0

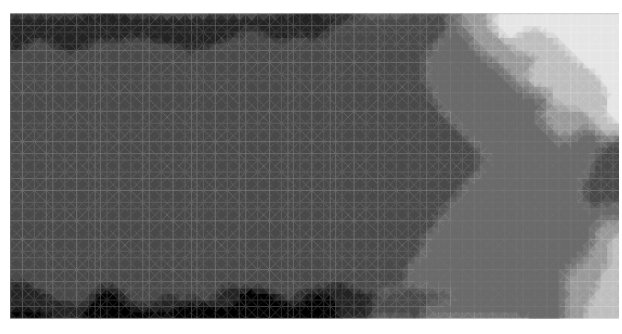

(c) Total density, iteration 100

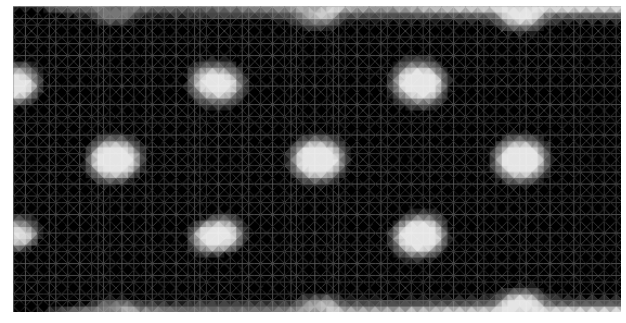

(b) Total density, iteration 50

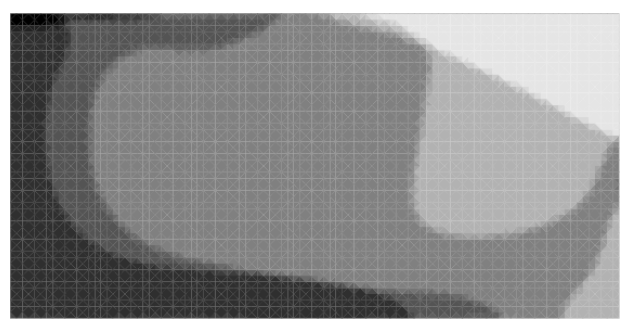

(d) Final total density (iteration 180)

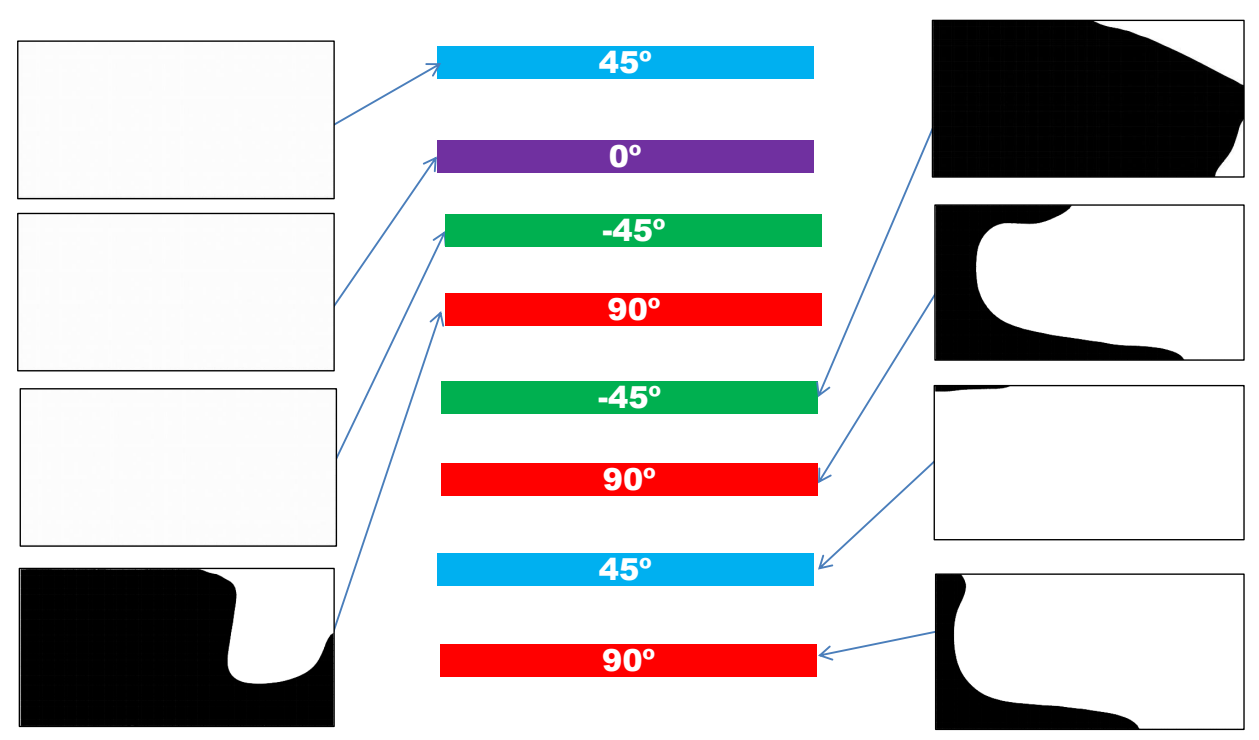

(e) Optimal stacking sequence and shape of each ply. The outer layers are upper in the figure.

Figure 10: Numerical results for the test case of Subsection 5.5 under the first load case (local vertical force) for $p=2$. (a),(b),(c),(d): Evolution of the total density. (e): Optimal stacking sequence and in-plane shape of each ply. 


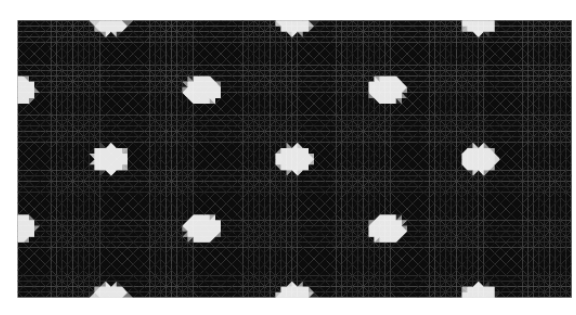

(a) Total density, iteration 0

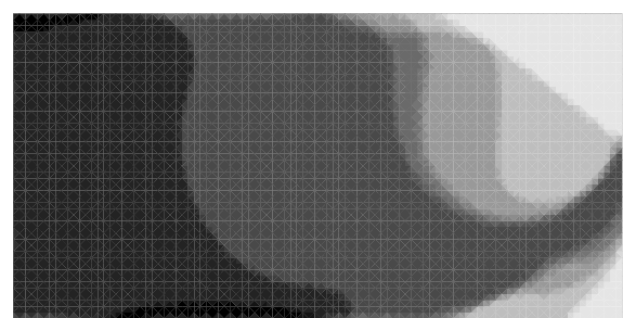

(c) Total density, iteration 100

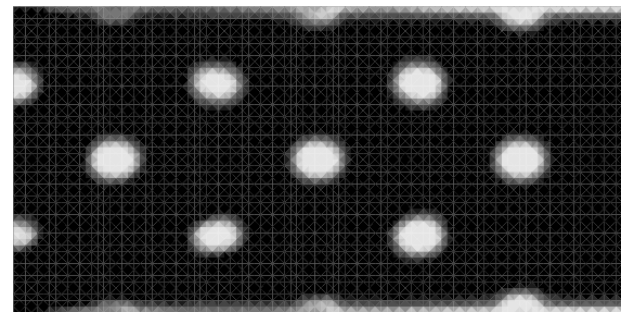

(b) Total density, iteration 50

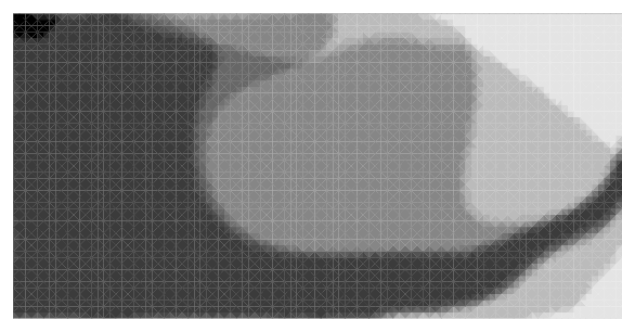

(d) Final total density (iteration 380)

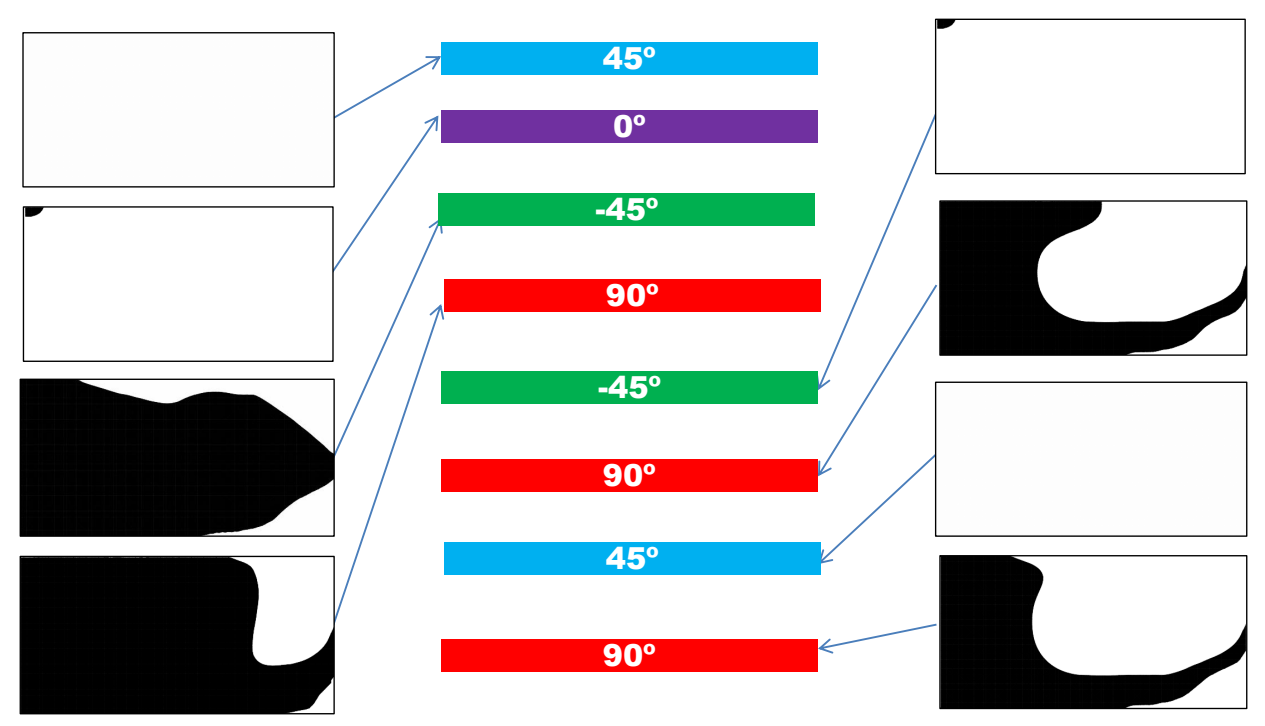

(e) Optimal stacking sequence and shape of each ply. The outer layers are upper in the figure.

Figure 11: Numerical results for the test case of Subsection 5.5 under the first load case (local vertical force) for $p=6$. (a),(b),(c),(d): Evolution of the total density. (e): Optimal stacking sequence and in-plane shape of each ply. 


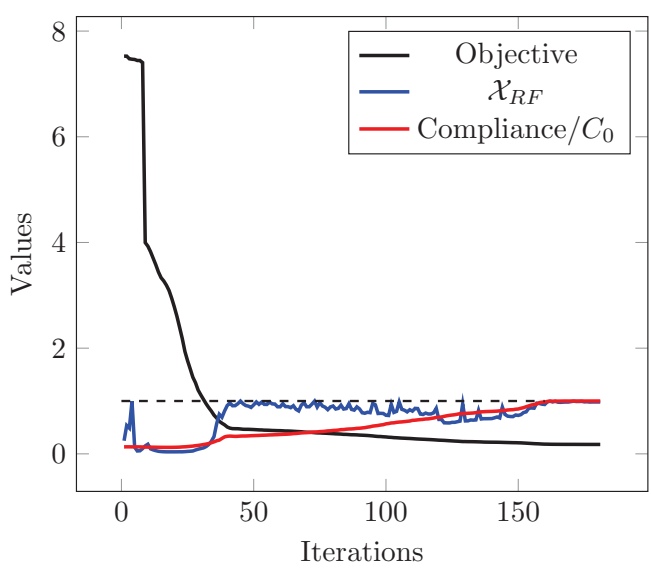

(a) $L^{p}$ approximation with $p=2$.

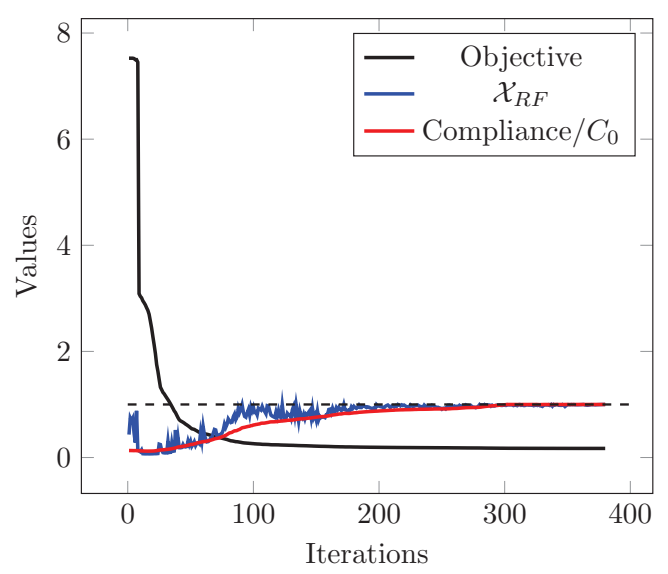

(b) $L^{p}$ approximation with $p=6$.

Figure 12: Convergence history for the test case of Subsection 5.5 under the first load case.

framework of the TOP3 project and also Dr. Stéphane Grihon and Vassili Srithammavahn from Airbus for many helpful discussions and a rich insight into industrial composite design. We are also thankful to Marc Albertelli and Jean-Leopold Vié for allowing us to use their adaptation of the method of feasible direction to the level set method [5]. This work was completed while G.D. was a PhD student working at the Airbus Group. G.A. is a member of the DEFI project at INRIA Saclay Ile-de-France.

\section{References}

[1] M. M. A. Catapano. A multi-scale approach for the optimum design of sandwich plates with honeycomb core. part i: homogenisation of core properties. Composite Structures, 118.

[2] M. M. A. Catapano. A multi-scale approach for the optimum design of sandwich plates with honeycomb core. part ii: the optimisation strategy. Composite Structures, 118.

[3] D. B. Adams, L. T. Watson, and Z. Gürdal. Blending of composite panel designs using genetic algorithms. In Parallel and Distributed Processing Symposium., Proceedings International, IPDPS 2002, Abstracts and CD-ROM, pages 6-pp. IEEE, 2001.

[4] D. B. Adams, L. T. Watson, Z. Gürdal, and C. M. Anderson-Cook. Genetic algorithm optimization and blending of composite laminates by locally reducing laminate thickness. Advances in Engineering Software, 35(1):35-43, 2004.

[5] M. Albertelli and J.-L. Vié. personal communication.

[6] G. Allaire. Conception optimale de structures, volume 58 of Mathématiques et Applications. Springer, 2006.

[7] G. Allaire, C. Dapogny, G. Delgado, and G. Michailidis. Multiphase structural optimization via a level set method. Control, Optimization and Calculus of Variations, 20:576-611, 2014.

[8] G. Allaire, F. Jouve, and A.-M. Toader. Structural optimization using sensitivity analysis and a level-set method. Journal of computational physics, 194(1):363-393, 2004.

[9] L. Ambrosio and G. Buttazzo. An optimal design problem with perimeter penalization. Calculus of Variations and Partial Differential Equations, 1(1):55-69, 1993.

[10] C. Antonio, A. T. Marques, and A. V. Soeiro. Optimization of laminated composite structures using a bilevel strategy. Composite structures, 33(4):193-200, 1995.

[11] M. Bendsøe and O. Sigmund. Topology optimization: Theory, methods and applications. Springer, Berlin, 2003. 


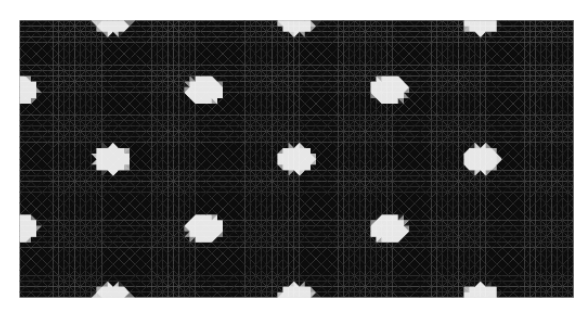

(a) Total density, iteration 0

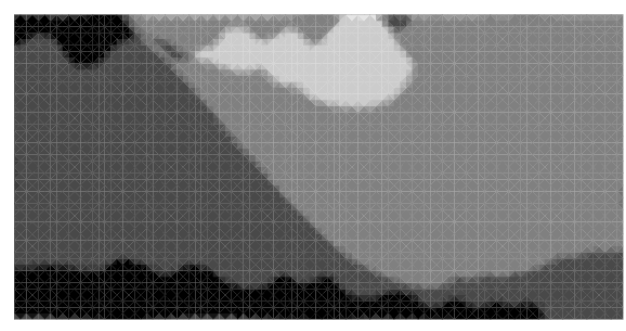

(c) Total density, iteration 50

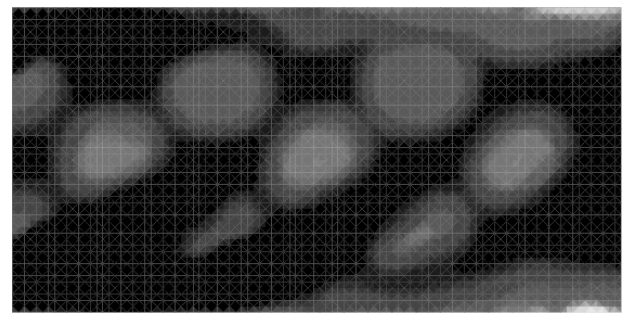

(b) Total density, iteration 20

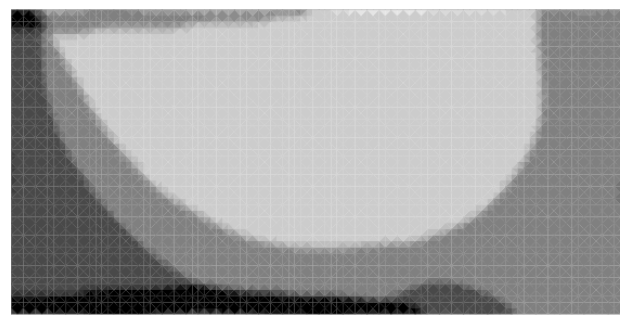

(d) Final total density (iteration 95)

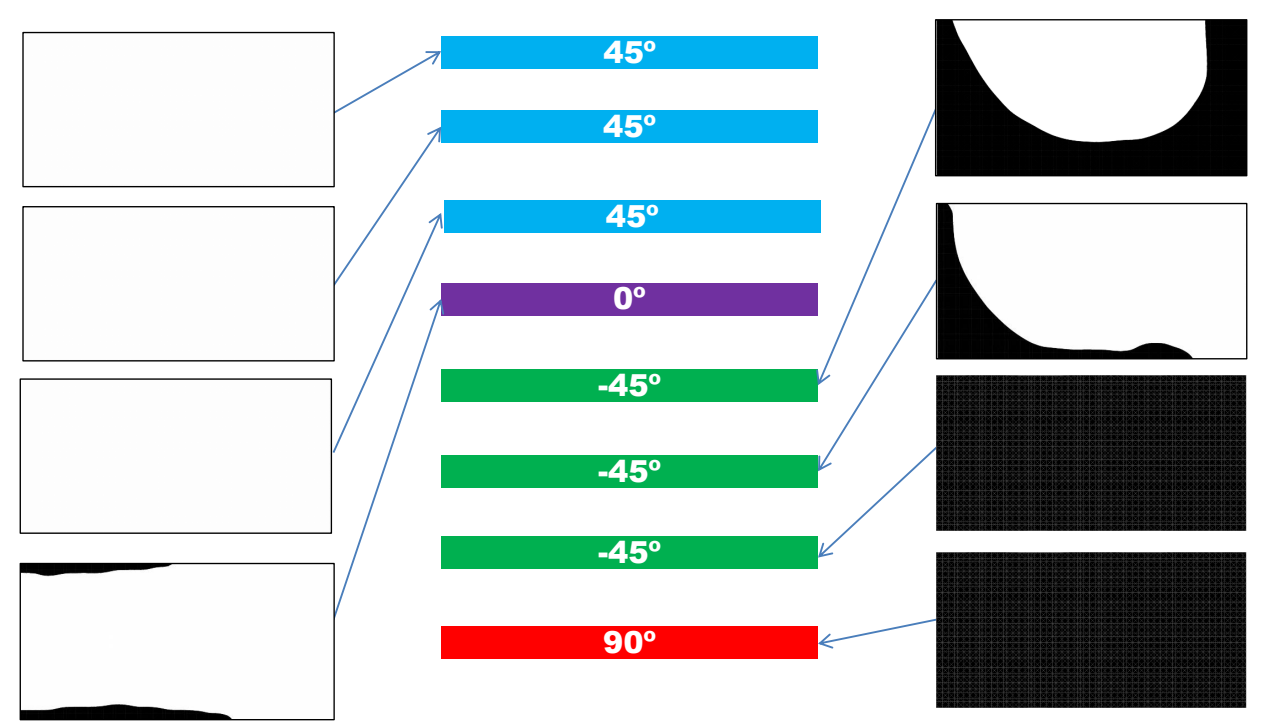

(e) Stacking sequence and shape of each ply. The outer layers are upper in the figure.

Figure 13: Numerical results for the test case of Subsection 5.5 under the second load case (uniform vertical force) for $p=6$ and variable layer density. (a),(b),(c),(d): Evolution of the total density. (e): Optimal stacking sequence and in-plane shape of each ply. 


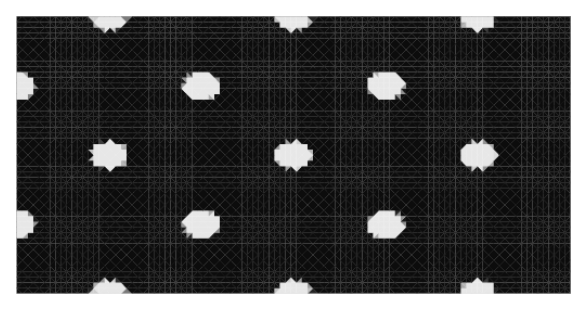

(a) Total density, iteration 0

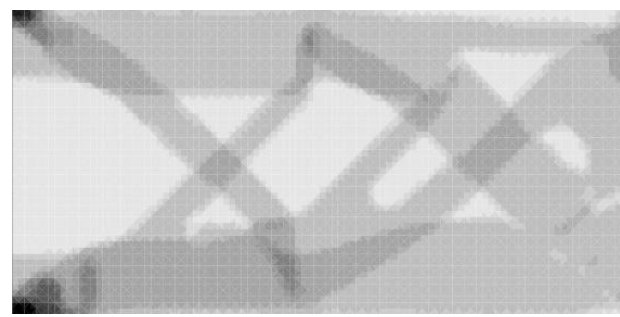

(c) Total density, iteration 50

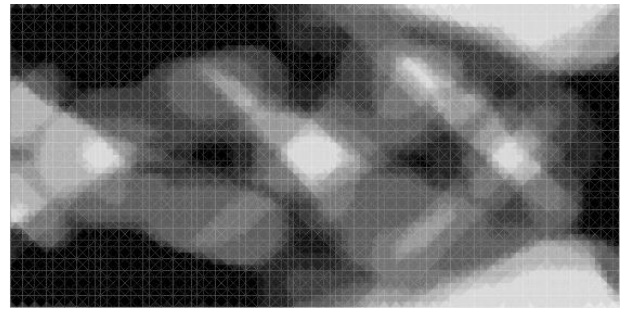

(b) Total density, iteration 20

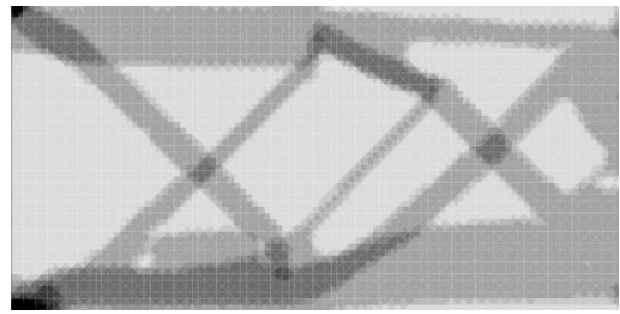

(d) Final total density (iteration 62)

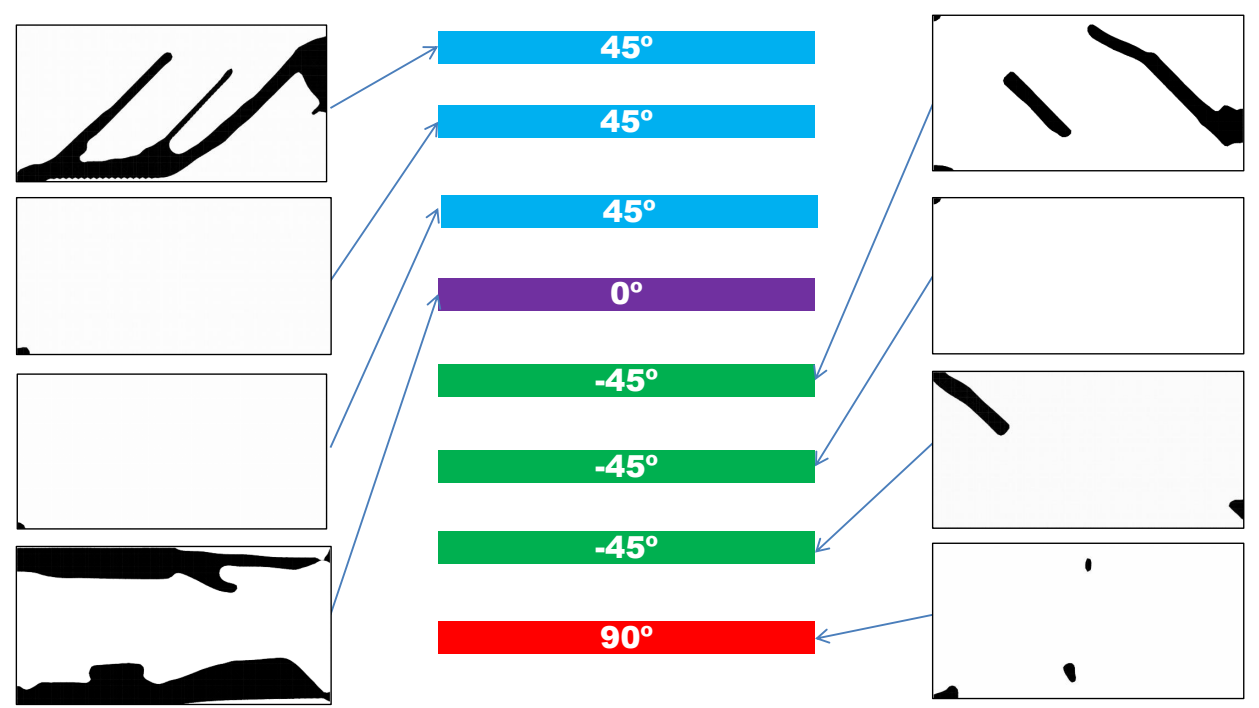

(e) Stacking sequence and shape of each ply. The outer layers are upper in the figure.

Figure 14: Numerical results for the test case of Subsection 5.5 under the second load case (uniform vertical force) for $p=6$ and uniform layer density $\rho=1$. (a),(b),(c),(d): Evolution of the total density. (e): Optimal stacking sequence and in-plane shape of each ply. 


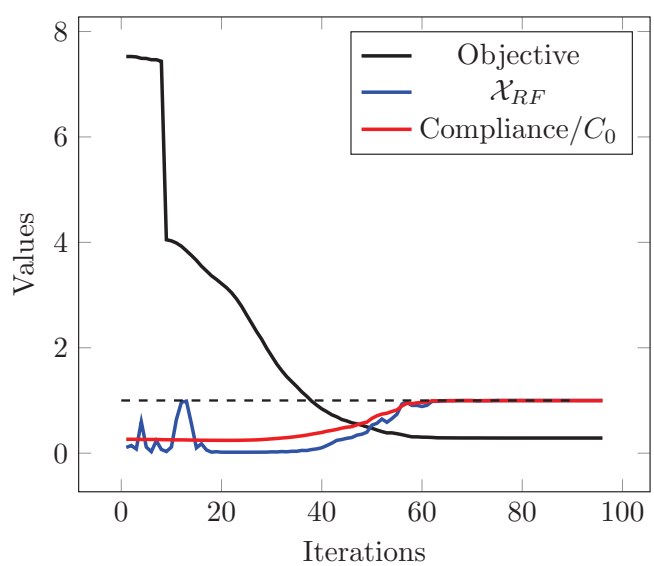

(a) Variable material densities (cf. Remark 5.7).

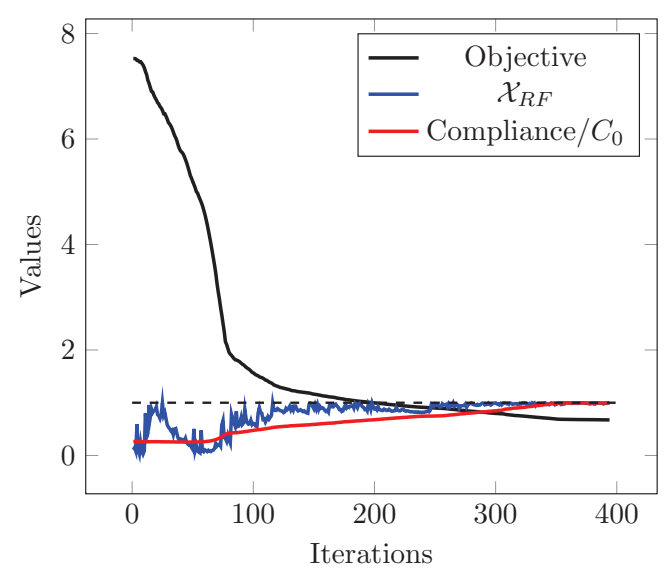

(b) Uniform material density $\rho=1$.

Figure 15: Convergence history for the test case of Subsection 5.5 under the second load case $\left(L^{p}\right.$ approximation with $p=6)$.

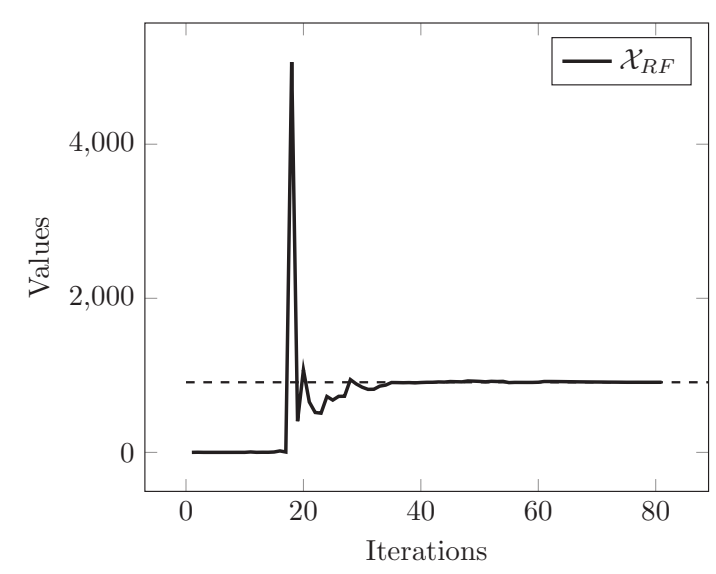

(a) Value of $\mathcal{X}_{R F}$.

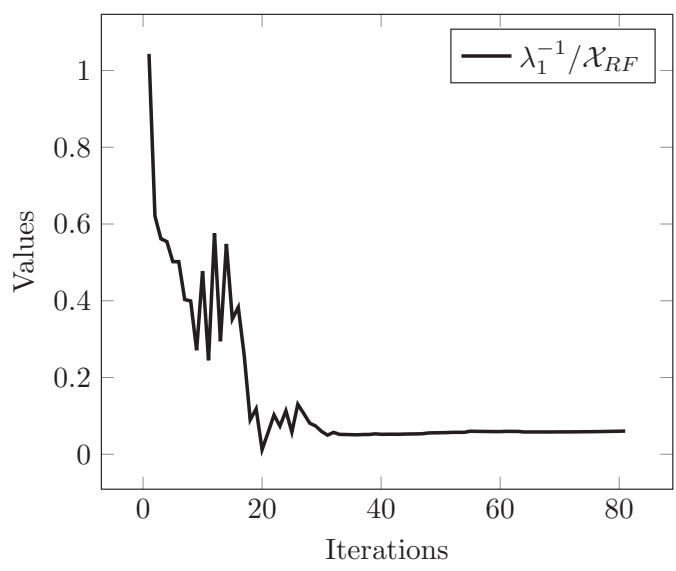

(b) Ratio $\lambda_{1}^{-1} / \mathcal{X}_{R F}$.

Figure 16: Comparison of $\lambda_{1}$ and $\lambda_{R F}$ : convergence history for the test case of Subsection 5.4 
[12] D. Bettebghor and N. Bartoli. Approximation of the critical buckling factor for composite panels. Struct. Multidiscip. Optim., 46(4):561-584, 2012.

[13] J. Blasques. Multi-material topology optimization of laminated composite beams with eigenfrequency constraints. Composite Structures, 111(1):45-55, 2014.

[14] J. Blasques and M. Stolpe. Multi-material topology optimization of laminated composite beam cross sections. Composite Structures, 94(11):3278-3289, 2012.

[15] F. Bloom and D. W. Coffin. Handbook of the Thin Plate Buckling and Post Buckling. CRC Press, 2001.

[16] M. Bruyneel. Sfp, a new parameterization based on shape functions for optimal material selection: application to conventional composite plies. Structural and Multidisciplinary Optimization, 43(1):1727, 2011.

[17] M. Bruyneel, S. Grihon, and M. Sosonkina. New approach for the stacking sequence optimization based on continuous topology optimization. 8th ASMO UK/ISSMO conference on engineering optimization, London, 2010.

[18] A. Carpentier. Optimisation multi-niveaux de panneaux composites. PhD thesis, Université de Toulouse, Université Toulouse III-Paul Sabatier, http://thesesups.upstlse.fr/234/1/CarpentierAlban.pdf, 2008.

[19] P. Ciarlet. Theory of Plates, Mathematical Elasticity Vol II, volume 27 of Studies in Mathematics and its applications. North Holland, 1997.

[20] P. G. Ciarlet and P. Rabier. Les équations de von Kármán. Number 826 in Lecture Notes in Mathematics. Springer Verlag, 1980.

[21] F. de Gournay. Velocity extension for the level-set method and multiple eigenvalues in shape optimization. SIAM J. Control Optim., 45(1):343-367, 2006.

[22] D. De Leon, C. de Souza, J. Fonseca, and R. da Silva. Aeroelastic tailoring using fiber orientation and topology optimization. Structural and Multidisciplinary Optimization, 46(5):663-677, 2012.

[23] G. Delgado. Optimization of composite structures. PhD thesis, Centre de Mathématiques Appliquées, École Polytechnique, 2014.

[24] S. Dempe. Foundations of bilevel programming. Springer, 2002.

[25] M. A. Duran and I. E. Grossmann. An outer-approximation algorithm for a class of mixed-integer nonlinear programs. Mathematical programming, 36(3):307-339, 1986.

[26] O. Erdal and F. O. Sonmez. Optimum design of composite laminates for maximum buckling load capacity using simulated annealing. Composite Structures, 71(1):45-52, 2005.

[27] R. Fletcher and S. Leyffer. Solving mixed integer nonlinear programs by outer approximation. Mathematical programming, 66(1-3):327-349, 1994.

[28] Freefem++. http://www.freefem.org/ff++/, 2013.

[29] M. Funnell. Targeting composite wing performance-optimum location of laminate boundaries. In Proceedings of Altair UK CAE Technology Conference 200\%, 2007.

[30] Z. Gürdal, R. T. Haftka, and P. Hajela. Design and optimization of laminated composite materials. Wiley. com, 1999.

[31] R. T. Haftka, Z. Gürdal, and M. Kamat. Elements of structural optimization, volume 11. Springer, 1992.

[32] R. T. Haftka and J. L. Walsh. Stacking-sequence optimization for buckling of laminated plates by integer programming. AIAA journal, 30(3):814-819, 1992.

[33] R. T. Haftka and L. T. Watson. Multidisciplinary design optimization with quasiseparable subsystems. Optimization and Engineering, 6(1):9-20, 2005. 
[34] F. Hecht. New development in freefem++. J. Numer. Math., 20(3-4):251-265, 2012.

[35] L. E. Henrichsen, S.R. and E. Lund. Robust buckling optimization of laminated composite structures using discrete material optimization considering "worst" shape imperfections. Thin-Walled Structures, 94.

[36] A. Henrot and M. Pierre. Variation et Optimisation des formes, Une analyse géométrique, volume 48 of Mathématiques et Applications. Springer, 2005.

[37] J. E. Herencia, R. T. Haftka, P. M. Weaver, and M. I. Friswell. Lay-up optimization of composite stiffened panels using linear approximations in lamination space. AIAA journal, 46(9):2387-2391, 2008.

[38] J. E. Herencia, P. M. Weaver, and M. I. Friswell. Optimization of long anisotropic laminated fiber composite panels with t-shaped stiffeners. AIAA journal, 45(10):2497-2509, 2007.

[39] C. F. Hvejsel, E. Lund, and M. Stolpe. Optimization strategies for discrete multi-material stiffness optimization. Structural and Multidisciplinary Optimization, 44(2):149-163, 2011.

[40] IBM-ILOG. Cplex. http://www-01.ibm.com/software/commerce/optimization/cplex-optimizer/.

[41] F.-X. Irisarri, D. H. Bassir, N. Carrere, and J.-F. Maire. Multiobjective stacking sequence optimization for laminated composite structures. Composites Science and Technology, 69(7):983-990, 2009 .

[42] R. Le Riche and R. T. Haftka. Optimization of laminate stacking sequence for buckling load maximization by genetic algorithm. AIAA journal, 31(5):951-956, 1993.

[43] D. Li and X. Sun. Nonlinear integer programming. Springer, 2006.

[44] C.-C. Lin and Y.-J. Lee. Stacking sequence optimization of laminated composite structures using genetic algorithm with local improvement. Composite structures, 63(3):339-345, 2004.

[45] E. Lindgaard and E. Lund. Nonlinear buckling optimization of composite structures. Computer methods in applied mechanics and engineering, 199(37):2319-2330, 2010.

[46] B. Liu and R. Haftka. Single-level composite wing optimization based on flexural lamination parameters. Structural and Multidisciplinary Optimization, 26(1-2):111-120, 2004.

[47] B. Liu, R. T Haftka, M. A Akgün, and A. Todoroki. Permutation genetic algorithm for stacking sequence design of composite laminates. Computer Methods in Applied Mechanics and Engineering, 186(2):357-372, 2000.

[48] D. Liu, V. V. Toroporov, O. M. Querin, and D. C. Barton. Bilevel optimization of blended composite wing panels. Journal of Aircraft, 48(1):107-118, 2011.

[49] W. Liu, R. Butler, and H. A. Kim. Optimization of composite stiffened panels subject to compression and lateral pressure using a bi-level approach. Structural and Multidisciplinary Optimization, $36(3): 235-245,2008$.

[50] W. Liu, R. Butler, A. R. Mileham, and A. J. Green. Bilevel optimization and postbuckling of highly strained composite stiffened panels. AIAA journal, 44(11):2562-2570, 2006.

[51] E. Lund. Buckling topology optimization of laminated multi-material composite shell structures. Composite Structures, 91(2):158-167, 2009

[52] P. V. M. Montemurro, A. Vincenti. A two-level procedure for the global optimum design of composite modular structuresapplication to the design of an aircraft wing. part 1: theoretical formulation. Journal of Optimization Theory and Applications, 155(1):1-23, 2012.

[53] P. V. M. Montemurro, A. Vincenti. A two-level procedure for the global optimum design of composite modular structuresapplication to the design of an aircraft wing. part 2: numerical aspects and examples. Journal of Optimization Theory and Applications, 155(1):24-53, 2012.

[54] MATLAB. version 8.0 (R2012b). The MathWorks Inc., Natick, Massachusetts, 2012. 
[55] L. Mesquita and M. P. Kamat. Optimization of stiffened laminated composite plates with frequency constraints. Engineering Optimization, 11(1-2):77-88, 1987.

[56] M. Miki and Y. Sugiyamat. Optimum design of laminated composite plates using lamination parameters. AIAA journal, 31(5):921-922, 1993.

[57] B. Mohammadi and O. Pironneau. Applied shape optimization for fluids, volume 28. Oxford University Press Oxford, 2001.

[58] E. Muñoz and M. Stolpe. Generalized Benders' decomposition for topology optimization problems. J. Global Optim., 51(1):149-183, 2011.

[59] S. Nagendra, R. T. Haftka, and Z. Gürdal. Stacking sequence optimization of simply supported laminates with stability and strain constraints. AIAA journal, 30(8):2132-2137, 1992.

[60] S. Nagendra, D. Jestin, Z. Gürdal, R. T. Haftka, and L. T. Watson. Improved genetic algorithm for the design of stiffened composite panels. Computers $\&$ Structures, 58(3):543-555, 1996.

[61] B. Niu, N. Olhoff, E. Lund, and G. Cheng. Discrete material optimization of vibrating laminated composite plates for minimum sound radiation. International Journal of Solids and Structures, 47(16):2097-2114, 2010.

[62] I. Nowak. Relaxation and decompostion methods for mixed integer nonlinear programming, volume 152. Birkhäuser Basel, 2005.

[63] S. Osher and J. Sethian. Fronts propagating with curvature-dependent speed: algorithms based on hamilton-jacobi formulations. Journal of computational physics, 79(1):12-49, 1988.

[64] J. N. Reddy. Mechanics of laminated composite plates: theory and analysis, volume 1. CRC press Boca Raton, 1997.

[65] B. Rousselet and D. Chenais. Continuité et différentiabilité d'éléments propres: application à l'optimisation de structures. Applied Mathematics and Optimization, 22(1):27-59, 1990.

[66] O. Seresta, Z. Gürdal, D. B. Adams, and L. T. Watson. Optimal design of composite wing structures with blended laminates. Composites Part B: Engineering, 38(4):469-480, 2007.

[67] J. Sethian. Level set methods and fast marching methods: evolving interfaces in computational geometry, fluid mechanics, computer vision, and materials science, volume 3. Cambridge university press, 1999.

[68] J. Sobieszczanski-Sobieski and R. T. Haftka. Multidisciplinary aerospace design optimization: survey of recent developments. Structural optimization, 14(1):1-23, 1997.

[69] J. Sokolowski and J.-P. Zolésio. Introduction to shape optimization. Springer, 1992.

[70] G. Soremekun, Z. Gürdal, R. Haftka, and L. Watson. Composite laminate design optimization by genetic algorithm with generalized elitist selection. Computers 6 Structures, 79(2):131-143, 2001.

[71] G. Soremekun, Z. Gürdal, C. Kassapoglou, and D. Toni. Stacking sequence blending of multiple composite laminates using genetic algorithms. Composite Structures, 56(1):53-62, 2002.

[72] S. Sorensen and E. Lund. Topology and thickness optimization of laminated composites including manufacturing constraints. Structural and Multidisciplinary Optimization, 48(2):249-265, 2013.

[73] S. R. Sorensen, S.N. and E. Lund. Dmtoa method for discrete material and thickness optimization of laminated composite structures. Structural and Multidisciplinary Optimization, 50(1):25-47, 2014.

[74] J. H. Starnes Jr and R. T. Haftka. Preliminary design of composite wings for buckling, strength, and displacement constraints. Journal of Aircraft, 16(8):564-570, 1979.

[75] J. Stegmann and E. Lund. Discrete material optimization of general composite shell structures. International Journal for Numerical Methods in Engineering, 62(14):2009-2027, 2005.

[76] A. Todoroki and M. Sasai. Stacking sequence optimizations using ga with zoomed response surface on lamination parameters. Advanced Composite Materials, 11(3):299-318, 2002. 
[77] D. Topkis and A. Veinott Jr. On the convergence of some feasible direction algorithms for nonlinear programming. SIAM Journal on Control, 5(2):268-279, 1967.

[78] J. van Campen, O. Seresta, M. M. Abdalla, and Z. Gürdal. General blending definitions for stacking sequence design of composite laminate structure. 49th AIAA/ASME/ASCE/AHS/ASC strutures, structural dynamics and material conference, Schaumburg, 2008.

[79] V. G. Vannucci P. A special class of uncoupled and quasi-homogeneous laminates. Compos Sci Technol, 61 .

[80] M. Y. Wang, X. Wang, and D. Guo. A level set method for structural topology optimization. Computer methods in applied mechanics and engineering, 192(1):227-246, 2003.

[81] X. Yuan, S. Zhang, L. Pibouleau, and S. Domenech. Une méthode d'optimisation non linéaire en variables mixtes pour la conception de procédés. RAIRO-Operations Research-Recherche Opérationnelle, $22(4): 331-346,1988$.

[82] M. Zhou, R. Fleury, and M. Kemp. Optimization of composite-recent advances and application. In 13th AIAA/ISSMO Multidisciplinary Analysis Optimization Conference, Fort Worth, Texas. September, pages 13-15, 2010. 